\title{
Basic interfacial thermodynamics and related mathematical background
}

\author{
Győző G. Láng1
}

Received: 31 May 2015/Accepted: 11 August 2015/Published online: 8 September 2015

(C) Springer International Publishing 2015

\begin{abstract}
This study is intended to give the reader a brief overview of the mathematical background to the thermodynamic theory of surfaces and interfaces. Some general aspects of the thermodynamics of systems with interfaces are discussed, and a concise treatment of interfaces within the framework of classical thermodynamics is provided. First, to introduce the reader to the topic, a reasonably simple thermodynamic treatment of interfaces, together with a brief description of the models widely used in the literature, is presented, the characteristics of the Gibbs "dividing plane" model and the Guggenheim "interphase" model are outlined, and a derivation of the Gibbs adsorption equation is given. In the subsequent sections, several important mathematical concepts (e.g., theory of homogeneous functions and partly homogeneous functions, Euler's theorem and the GibbsDuhem equation, Legendre transformation) and various functional relationships of the thermodynamics of surfaces and interfaces are summarized, with particular attention to some alternative formulations. Some of the mathematical principles and methods are explained using illustrative examples.
\end{abstract}

Keywords Interfacial thermodynamics - Gibbs model · Guggenheim model · Homogeneous functions · Partly homogeneous functions · Euler's theorem - Legendre transformation - Gibbs-Duhem equation

Electronic supplementary material The online version of this article (doi:10.1007/s40828-015-0015-z) contains supplementary material, which is available to authorized users.

Győző G. Láng

langgyg@chem.elte.hu

1 Institute of Chemistry, Laboratory of Electrochemistry and Electroanalytical Chemistry \& Department of Physical Chemistry, Eötvös Loránd University, Budapest Pázmány P. s. 1/A, Budapest 1117, Hungary

\section{Introduction}

Despite the advent of surface-sensitive techniques, thermodynamic measurements remain a valuable tool for the investigation of surfaces and interfaces.

Many (heterogeneous) systems of practical importance can only be described by complex thermodynamic models including interface(s). For instance, electrodes are, in fact, capillary systems, because the interactions between the different phases take place via the surface region. Thus, the understanding of the thermodynamics of these interfaces is of importance to all surface scientists and electrochemists.

The aim of the present study is to give a brief overview of the mathematical background, some important mathematical concepts, and various functional relationships underlying the thermodynamic theory of interfaces (theory of homogeneous functions and partly homogeneous functions, Euler's theorem and the Gibbs-Duhem equation, Legendre transformation). The mathematical principles are explained and illustrated with some typical examples.

First, to introduce the reader to the topic, a reasonably simple thermodynamic treatment of interfaces, together with a brief description of the models widely used in the literature, is presented (more detailed discussions can be found in several reviews and research papers [1-16]).

\section{Basic concepts and notions related to the thermodynamics of interfaces}

\section{Models of the interfacial region}

Interfacial thermodynamics is the study of the application of thermodynamics to interfacial phenomena, addressing topics, including adsorption, interfacial energies, interfacial 
tension, and superficial charge, and about relations among them [see e.g., 1-18]. Adsorption of one or more of the components, at one or more of the phase boundaries of a multicomponent, multiphase system, is said to occur if the concentrations in the interfacial layers are different from those in the adjoining bulk phases. Consequently, the overall stoichiometry of the system deviates from that corresponding to a reference system of (hypothetical) homogeneous bulk phases whose volumes and/or amounts are defined by suitably chosen dividing surfaces, or by a suitable algebraic method (see later).

The classic work is that of Gibbs [19]; a paper by Guggenheim and Adam [20] discusses the physical interpretation of surface excesses, and Guggenheim [21] has given a good summary of interfacial thermodynamics emphasizing a viewpoint somewhat different from that of Gibbs.

In many studies, the plane ideally marking the boundary between two phases is called the interface. Although interfaces are always dealt with from a thermodynamic point of view, if attention is actually focused on only one of the two phases, the plane marking the boundary between the phase and the environment is often called the surface of the phase (see e.g., [22]). The two words (i.e., "interface" and "surface") are often used synonymously, although interface is preferred for the boundary between two condensed phases and in cases where the two phases are named explicitly, e.g., the solid/gas interface [23].

The region between two phases where the properties vary between those in the bulk is the "interfacial" or "interface" region, and sometimes it is called the "interphase."

An interface or interphase does not exist in isolation, and valid thermodynamic conclusions can only be drawn by considering the system, namely the interface and the two bordering regions, as a whole. Provided that the radius of curvature is large, the interface/interphase may be regarded as a plane and its energy then differs from that of a bulk phase by a term expressing the contribution of changes of energy due to a change of the area of contact. Edge effects can be eliminated by considering a section of an interface in a larger system. There is no clear boundary between the interfacial region and the bulk of the phases, so that the thickness of the interphase depends on the model chosen to describe this region. The geometric area is represented by the product of the length and breadth of a rectangle enclosing part of a surface. Many properties of a system, for example, concentration of a particular species, vary as a function of the distance perpendicular to the surface, as shown in Fig. 1.

The classical Gibbs approach is based on a model in which a real interface layer is replaced by a dividing surface [19]. Gibbs found it mathematically convenient to consider an idealized system depicted in Fig. 1b, with properties identical with those of the whole real system. The "surface of discontinuity" or "dividing surface" in the idealized system is a two-dimensional region whose position is determined by the requirements that the property under consideration should maintain a uniform value in each bulk phase right up to the dividing surface. This corresponds to equating the two shaded areas in Fig. 1b. A disadvantage of this approach is that the position of the dividing surface alters according to the property considered.

In the Guggenheim model, two dividing surfaces, one at each boundary, are employed (Fig. 1c). It is assumed that there is a "surface" or "interfacial" layer of finite thickness $(\tau)$ bounded by two appropriately chosen surfaces parallel to the phase boundary, one in each of the adjacent homogeneous bulk phases. A layer of this kind is sometimes called a Guggenheim layer or "interphase." A disadvantage is that terms dependent on surface volume are present in the equations, but it is difficult to assign values to these terms. (It should be noted that for very highly curved surfaces, i.e., when the radius of curvature is of the same magnitude as $\tau$, the notion of a surface layer may lose its usefulness.)

Given a system, subsystems consisting of a segment of the interface and finite volumes of the adjacent phases can be selected. In principle, these subsystems should not be geometrically regular in shape; however, the rectangular parallelepiped-shaped domain is usually the most expedient selection. In two dimensions, the macroscopic subsystem selected for investigation is represented by the ABCD rectangle (Fig. 1).

Usually, the thickness of the interface or local values of physical quantities (parameters) cannot be measured. That is the reason why integrated quantities (which are accessible experimentally, or can be calculated from experimental data) are used for the thermodynamic characterization of interfaces. Generally, these quantities are given by the expression:

$\Psi^{\sigma}=\int_{\alpha \alpha}^{\beta \beta} Y(\xi) \mathrm{d} \xi$,

where $\xi$ is the coordinate perpendicular to the plane of the interface, $Y$ is the function of $\xi, \Psi^{\sigma}$ is the integrated physical quantity, and $\alpha \alpha$ and $\beta \beta$ are the two adjacent (homogeneous) phases.

Let the area of the surface or interface in the system defined according to the above concepts be denoted by $A$, and the internal energy by $U$. The volume $V$ of the system is the sum of the volumes of the two phases $\alpha \alpha$ and $\beta \beta$, and the volume of the inhomogeneous region is as follows: 
(a) real system
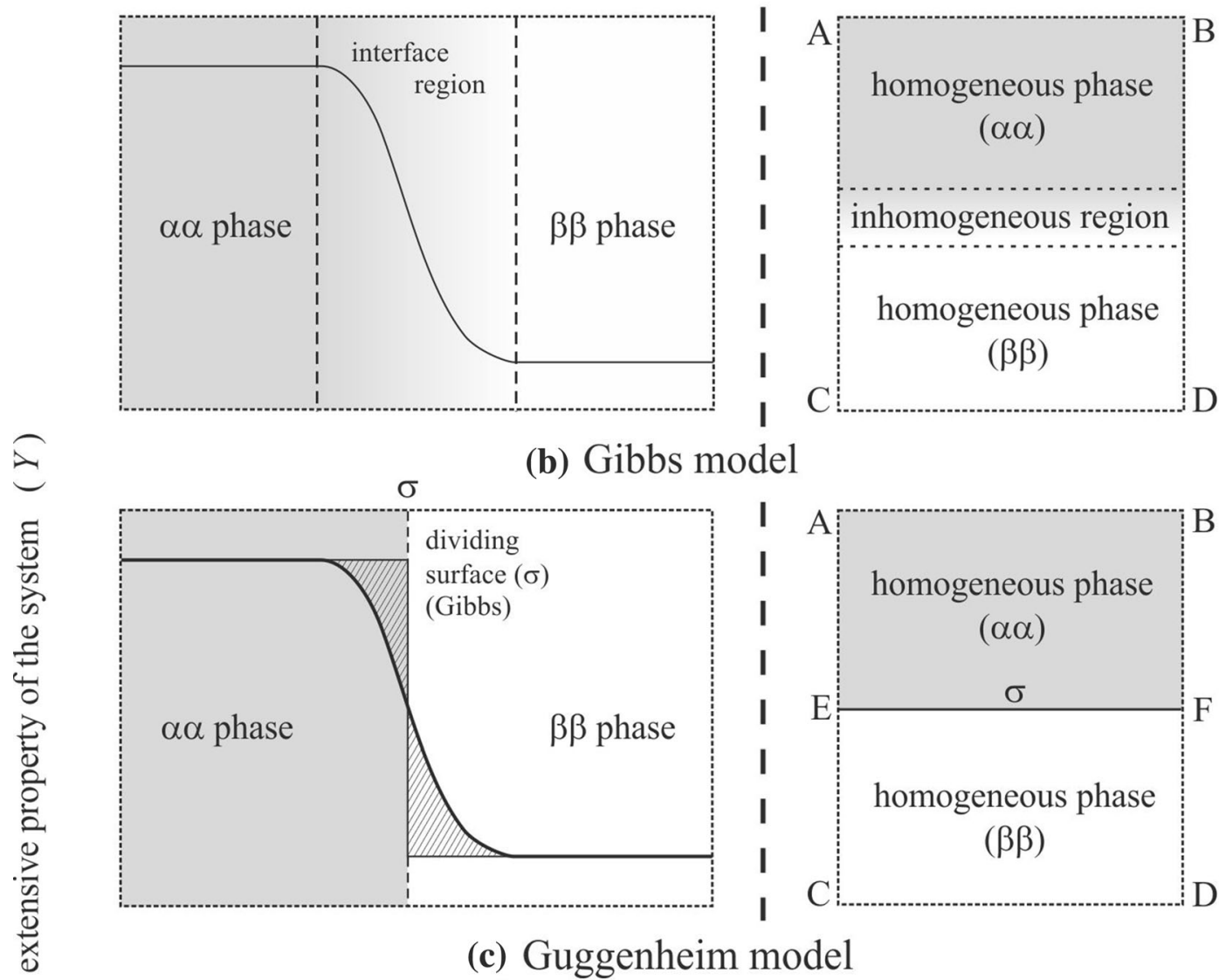

(b) Gibbs model
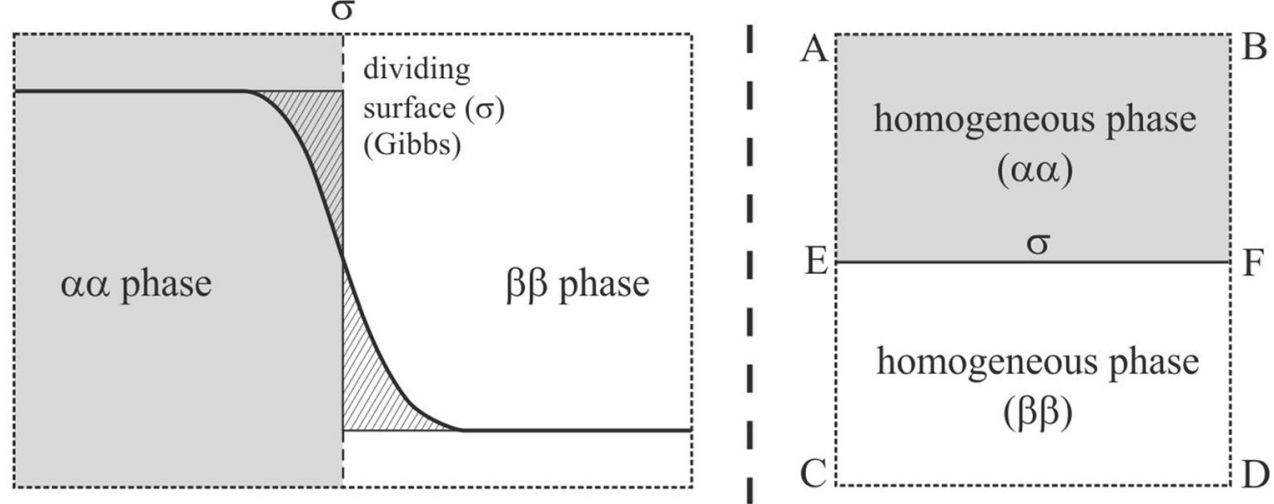

(c) Guggenheim model
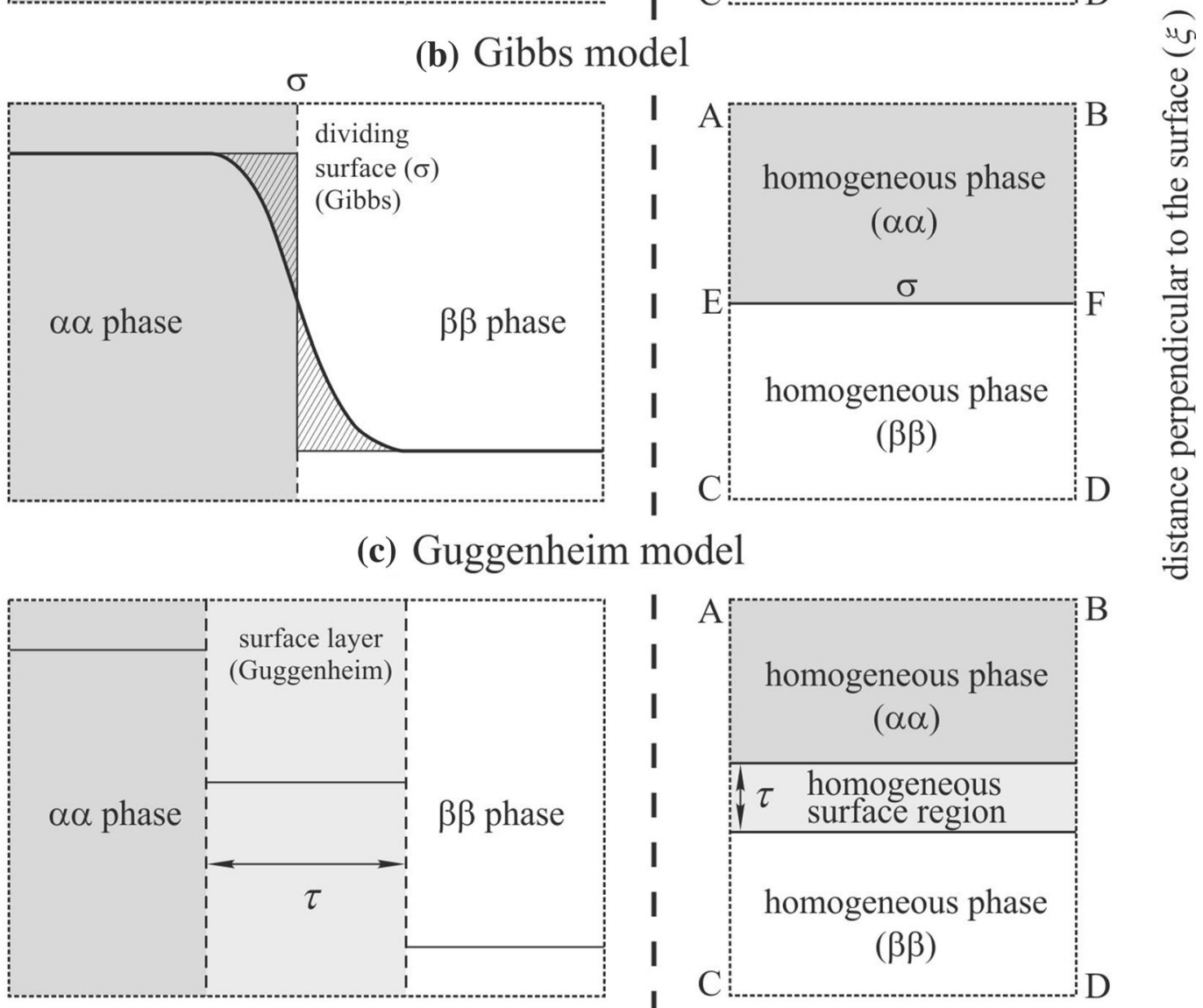

\section{distance perpendicular to the surface $(\xi)$}

Fig. 1 A schematic representation of the interfacial region, the Gibbs "dividing surface" ("surface of discontinuity" or "mathematical plane") and the "interfacial layer" concept ("interphase") proposed by Guggenheim. a The real system, $\mathbf{b}$ the Gibbs model of the

$V=V^{\alpha \alpha}+V^{\beta \beta}+V^{\mathrm{inh}}$.

The internal energy can be given as

$U=U^{\alpha \alpha}+U^{\beta \beta}+U^{\mathrm{inh}}$.

Of course, this division is completely arbitrary, since the values on the right-hand sides of Eqs. (2) and (3) depend on interface, and $\mathbf{c}$ the Guggenheim model of the interface. On the righthand side: the macroscopic subsystems selected for investigation are represented by the $\mathrm{ABCD}$ rectangles

the (arbitrary) choice of the dividing surface(s). In the Guggenheim model, the volume $V^{\sigma}$ of the interfacial layer is $V^{\sigma}=\tau A$.

The Gibbs dividing surface (or Gibbs surface) is a geometrical surface chosen parallel to the interface and used to define the volumes of the bulk phases. That is 
$V=V^{\alpha \alpha}+V^{\beta \beta}$

This means that the volume of the "surface phase is" $V^{\sigma} \equiv 0$.

\section{Adsorption}

As already discussed above, the Gibbs interface is a twodimensional homogeneous phase without thickness (i.e., the interface is regarded as a mathematical dividing surface). In Guggenheim's approach, the interface is considered to be a surface phase with finite thickness and volume treated in a way analogous to bulk phases, except that the thermodynamic equations contain terms related to the contributions of changes of energy due to changes of area and electrical state of the interface.

The two apparently different approaches can be essentially characterized by the following procedure:

a) There is an idealized surface or surface phase separating two homogeneous bulk phases (see Fig. 1). The bulk phases are in equilibrium with the surface phase.

b) Two separated reference systems $\alpha$ and $\beta$ thought to be noninteracting homogeneous bulk phases have to be chosen (see Fig. 2), the conditions of temperature, pressure, and composition being identical to those in the adsorption equilibrium. Both reference phases consist of suitably defined amounts of the components. Each of the chosen reference amounts is characterized by its respective molar or specific properties.

c) Any extensive property of the reference systems is simply the sum of the contributions from the reference amounts, without any contributions from interactions with the interfacial layer in the real system.

The surface excess quantities are then the respective differences between the real system and the chosen reference systems (or reference phases). As explained above, this can be done, e.g., in the Gibbs sense of the total extensive quantity minus its amount residing in hypothetical bulk phases that are uniform up to a mathematical dividing surface, or in the Guggenheim sense of excesses over the average bulk amount in a boundary zone ("surface phase") of finite but small thickness. Obviously, if the Gibbs model is used for the definition of surface excesses, the reference amounts in the two reference phases are thought to be contained in and making up the volume of the actual real system, but can equally well be thought to be quite independent and spatially apart one from the other. On the other hand, however, the volume of the chosen

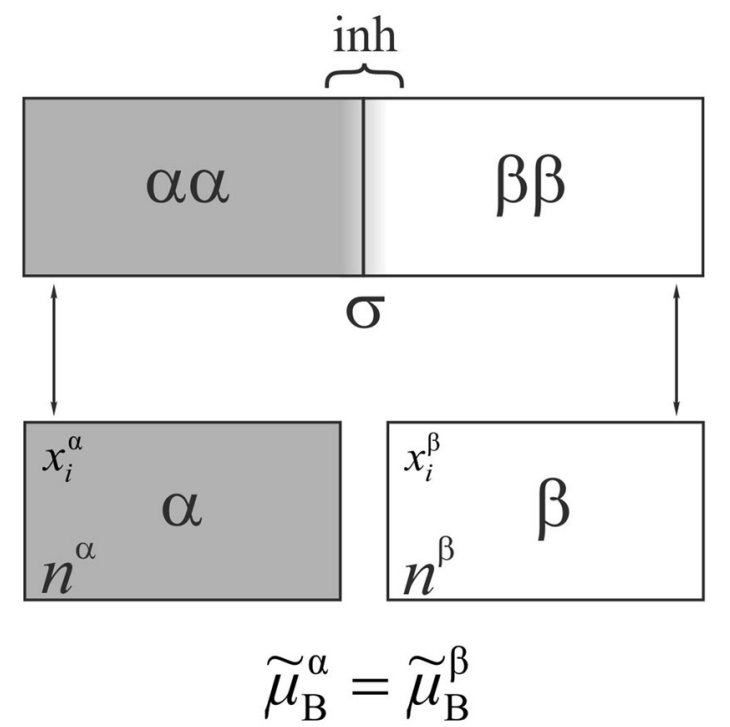

Fig. 2 Scheme of the "real system" with the inhomogeneous "interfacial region" (inh) and the "model system." In the Gibbsian model, there is a hypothetical "dividing surface" $(\sigma)$ (in the Guggenheim model a hypothetical, three-dimensional surface phase, see Fig. 1) which is separating two homogeneous bulk phases $\alpha \alpha$ and $\beta \beta$ (that are in equilibrium with the surface region). The two reference systems are $\alpha$ and $\beta$

reference amounts is not necessarily equal to the volume of the real system. It is even not necessary that the corresponding phases are effectively present in their chosen reference states within the real system. In principle, this is why the Gibbs and the Guggenheim approaches can be considered as equivalent. Nevertheless, there is an important restriction in the Guggenheim approach replacing the condition of equivalent volumes in the Gibbs method: the reference systems must be chosen in such a manner that the remaining "surface phase" has a constant thickness. Thus, this restriction essentially affects the choice of the geometrical shape of the reference systems. However, since the reference systems are homogeneous bulk phases, their thermodynamic properties are independent of the shape. For this reason, a set of appropriate reference systems can be always selected without loss of generality. This consideration determines implicitly the selection of thermodynamic systems "with cylindrical shape" [24], a "parallelepiped" [25], or simply as a "section" of the interface cut out by perpendicular planes [26-28].

The surface excess amount or Gibbs adsorption of component $i$ is $n_{i}^{\sigma}$, which may be positive or negative, and is defined as the excess of the amount of this component actually present in the system over that present in a reference system of the same volume as the real system and in which the bulk concentrations in the two phases remain uniform up to the Gibbs dividing surface. 


$$
\begin{aligned}
n_{i}^{\sigma} & =n_{i}-n_{i}^{\alpha}-n_{i}^{\beta}=n_{i}-n^{\alpha} x_{i}^{\alpha}-n^{\beta} x_{i}^{\beta} \\
& =n_{i}-n^{\alpha} x_{i}^{\alpha \alpha}-n^{\beta} x_{i}^{\beta \beta}
\end{aligned}
$$

where $n_{i}$ is the total amount of component $i$ in the "real" system, $x_{i}^{\alpha}$ and $x_{i}^{\beta}$ are the mole fractions in phases $\alpha$ and $\beta$, respectively, and $n^{\alpha}$ and $n^{\beta}$ are the total amounts of the components ("total number of moles") in the reference systems. It is clear from Eq. (6) that the surface excess amount is well defined only when $n^{\alpha}$ and $n^{\beta}$ are fixed. It can be also seen that with different $n^{\alpha}$ and $n^{\beta}$ values we have different values for $n_{i}^{\sigma}$.

According to the above considerations, the surface excess $X^{\sigma}$ of any extensive property $X$ is calculated as

$X^{\sigma}=X-X^{\alpha}-X^{\beta}$,

where $X$ denotes the value of the extensive property for the whole system and $X^{\alpha}$ and $X^{\beta}$ are the values for the reference systems.

The relation that gives the internal energy $U$ as a function of the extensive parameters is a fundamental relation. If the fundamental relation of a particular system is known, all conceivable thermodynamic information about this system can be ascertained [29]. The internal energies of the reference phases are given by

$U^{\alpha}=U^{\alpha}\left(S^{\alpha}, V^{\alpha}, n_{1}^{\alpha} \ldots n_{m}^{\alpha}\right)$

and

$U^{\beta}=U^{\beta}\left(S^{\beta}, V^{\beta}, n_{1}^{\beta} \ldots n_{m}^{\beta}\right)$.

The internal energy $(U)$ of the system depends on the entropy $(S)$, volume $(V)$, amounts $n_{1} \ldots n_{m}$ of the components $1, \ldots m$, and the surface area $(A)$ :

$U=U\left(S, V, A, n_{1} \ldots n_{m}\right)$.

The excess of the internal energy is given by

$U^{\sigma}=U-U^{\alpha}-U^{\beta}$,

and the excess of the entropy is

$S^{\sigma}=S-S^{\alpha}-S^{\beta}$.

The excess internal energy function

$U^{\sigma}=U^{\sigma}\left(S^{\sigma}, V^{\sigma}, A, n_{1}^{\sigma} \ldots n_{m}^{\sigma}\right)$

is a homogeneous function of degree one with respect to all variables (see "Homogeneous functions" section, especially Examples \#1 and \#4), if $V^{\sigma} \equiv 0$ (Gibbs model), or $V^{\sigma}=A \tau$ (Guggenheim model), since it is evident that

$U^{\sigma}\left(k S^{\sigma}, k V^{\sigma}, k A, k n_{i}^{\sigma} \ldots k n_{m}^{\sigma}\right)=k U^{\sigma}\left(S^{\sigma}, V^{\sigma}, A, n_{i}^{\sigma} \ldots n_{m}^{\sigma}\right)$

for all $k>0$ real numbers.
Therefore, according to Euler's theorem (see "Homogeneous functions" section and Example \#4), and in the framework of the Gibbs model

$U^{\sigma}=T^{\sigma} S^{\sigma}+\gamma A+\sum_{i} \mu_{i}^{\sigma} n_{i}^{\sigma}$,

where $\gamma$ is the intensive (interfacial) parameter conjugate to the extensive variable $A$.

Due to the thermodynamic equilibrium

$T^{\sigma}=T^{\alpha}=T^{\beta}=T^{\alpha \alpha}=T^{\beta \beta}=T$,

and

$\mu_{i}^{\sigma}=\mu_{i}^{\alpha}=\mu_{i}^{\beta}=\mu_{i}^{\alpha \alpha}=\mu_{i}^{\beta \beta}=\mu_{i}$.

According to the equations like the two above, it is not necessary to use superscripts to distinguish $T, \mu_{1} \ldots \mu_{m}$, in the different equilibrium phases because these must have uniform values throughout $\alpha, \beta, \alpha \alpha, \beta \beta$, and $\sigma$ (due to the equilibrium assumptions).

In the two reference phases, the following relationships are valid:

$U^{\alpha}=T S^{\alpha}-p V^{\alpha}+\sum_{i} \mu_{i} n_{i}^{\alpha}$,

and

$U^{\beta}=T S^{\beta}-p V^{\beta}+\sum_{i} \mu_{i} n_{i}^{\beta}$.

According to Eq. (15), the intensive parameter $(\gamma)$ is defined by

$\gamma=\left(\frac{\partial U^{\sigma}}{\partial A}\right)_{S^{\sigma}, n_{1}^{\sigma} \ldots n_{m}^{\sigma}}$.

Although this expression is mathematically correct, it is not really useful for practical purposes. Equation (15) expresses the dependence of the energy $U$ on the basis of independent variables $S^{\sigma}, A, n_{1}^{\sigma} \ldots n_{m}^{\sigma}$. This set of independent variables is not by any means the most convenient. It is usually preferable to use $T$ as an independent variable instead of $S$. If the experiment is such that the external conditions are constant temperature and constant pressure, the most convenient potential function to use is the Gibbs free energy function, $G\left(T, p, n_{1} \ldots n_{m}\right)$, obtained from $U\left(S, V, n_{1} \ldots n_{m}\right)$ by two subsequent Legendre transformations (see "Adsorption" section and especially Example \#12):

$G^{\alpha}=U^{\alpha}+p V^{\alpha}-T S^{\alpha}$

and

$G^{\beta}=U^{\beta}+p V^{\beta}-T S^{\beta}$.

Consequently 
$G^{\alpha}=\sum_{i} \mu_{i} n_{i}^{\alpha}$

and

$G^{\beta}=\sum_{i} \mu_{i} n_{i}^{\beta}$.

The excess Gibbs free energy function is given as

$G^{\sigma}=\gamma A+\sum_{i} \mu_{i} n_{i}^{\sigma}$,

and $\gamma$ is defined by

$\gamma=\left(\frac{\partial G^{\sigma}}{\partial A}\right)_{T, n_{1}^{\sigma} \ldots n_{m}^{\sigma}}$.

Unfortunately, this definition of $\gamma$ is still not appropriate for experimental studies or to confirm experimental results since $G^{\sigma}\left(T, A, n_{1}^{\sigma} \ldots n_{m}^{\sigma}\right)$ remains ill-defined and arbitrary (because $n_{1}^{\sigma} \ldots n_{m}^{\sigma}$ clearly depend on the selection of the reference systems). The Gibbs free energy function for the whole system can be expressed as

$$
\begin{aligned}
G & =\gamma A+\sum_{i} \mu_{i} n_{i}^{\alpha}+\sum_{i} \mu_{i} n_{i}^{\beta}+\sum_{i} \mu_{i} n_{i}^{\sigma} \\
& =\gamma A+\sum_{i} \mu_{i}\left(n_{i}^{\alpha}+n_{i}^{\beta}+n_{i}^{\sigma}\right) .
\end{aligned}
$$

This means that $\gamma$ can also be defined in terms of the Gibbs free energy function of the whole system as

$\gamma=\left(\frac{\partial G}{\partial A}\right)_{T, p, n_{1} \ldots n_{m}}$,

or in terms of the Helmholtz (free) energy function as

$\gamma=\left(\frac{\partial F}{\partial A}\right)_{T, V, n_{1} \ldots n_{m}}$,

when the Helmholtz energy or "free energy" function is defined as the Legendre transform of the internal energy function $(F=U-T \cdot S)$. On the other hand-still remaining in the framework of the Gibbs model —it should be noted that since no volume term appears in Eq. (15), there is no distinction between the surface Helmholtz and Gibbs free energies.

According to the above discussions, $G^{\sigma}$ is a partly homogeneous function of degree one (see "The GibbsDuhem equation" section) in the variables $A$ and $n_{1}^{\sigma} \ldots n_{m}^{\sigma}$. The expression for the total differential of $G^{\sigma}$ is

$$
\begin{aligned}
\mathrm{d} G^{\sigma}= & \left(\frac{\partial G^{\sigma}}{\partial T}\right)_{A, n_{1}^{\sigma} \ldots n_{m}^{\sigma}} \mathrm{d} T+\left(\frac{\partial G^{\sigma}}{\partial A}\right)_{T, n_{1}^{\sigma} \ldots n_{m}^{\sigma}} \mathrm{d} A \\
& +\sum_{i}\left(\frac{\partial G^{\sigma}}{\partial A}\right)_{T, A, n_{j \neq i}^{\sigma}} \mathrm{d} n_{i}^{\sigma} .
\end{aligned}
$$

Taking into account that $\left(\frac{\partial G^{\sigma}}{\partial T}\right)_{A, n_{1}^{\sigma} \ldots n_{m}^{\sigma}}=-S^{\sigma},\left(\frac{\partial G^{\sigma}}{\partial A}\right)_{T, n_{1}^{\sigma} \ldots n_{m}^{\sigma}}$ $=\gamma$, and $\left(\frac{\partial G^{\sigma}}{\partial A}\right)_{T, A, n_{j \neq i}^{\sigma}}=\mu_{i}$, Equation (30) can be written as $\mathrm{d} G^{\sigma}=-S^{\sigma} \mathrm{d} T+\gamma \mathrm{d} A+\sum_{i} \mu_{i} \mathrm{~d} n_{i}^{\sigma}$.

The differential of Eq. (25) is

$\mathrm{d} G^{\sigma}=\gamma \mathrm{d} A+A \mathrm{~d} \gamma+\sum_{i} \mu_{i} \mathrm{~d} n_{i}^{\sigma}+\sum_{i} n_{i}^{\sigma} \mathrm{d} \mu_{i}$.

There are thus two (general) expressions for $\mathrm{d} G^{\sigma}$ (Eqs. 31 and 32), both of which are correct. This can only be the case if

$S^{\sigma} \mathrm{d} T+A \mathrm{~d} \gamma+\sum_{i} n_{i}^{\sigma} \mathrm{d} \mu_{i}=0$.

Equation (33) is the so-called Gibbs-Duhem equation for interfaces (see "The Gibbs-Duhem equation" section). At constant temperature

$-A \mathrm{~d} \gamma=\sum_{i} n_{i}^{\sigma} \mathrm{d} \mu_{i}$.

Dividing both sides of Eq. (33) by $A$ yields

$-\mathrm{d} \gamma=\sum_{i} \frac{n_{i}^{\sigma}}{A} \mathrm{~d} \mu_{i}=\sum_{i} \Gamma_{i} \mathrm{~d} \mu_{i}$,

where $\Gamma_{i}$ is the surface excess concentration of species $i$. Equation (35) is commonly called the Gibbs adsorption equation.

In the case of liquid/liquid interfaces, the interfacial intensive parameter $(\gamma)$ can be identified with the interfacial tension. (Note that in case of solid/liquid interfaces there is some controversy in the literature concerning the correct name of $\gamma$, e.g., it is sometimes called "specific surface energy" or "surface stress" [1, 16, 26, 30, 31]).

There are two important points that should be addressed here:

1. In the case of ionic components (charged species), "electrochemical potentials" $\left(\tilde{\mu}_{i}\right)$ may be used instead of "chemical potentials" in the corresponding equations.

2. It follows from Eq. (6) (which is the definition equation of the surface excess amounts) that the $\Gamma_{i}$ values are uncertain, since they depend on the arbitrary selection of $n^{\alpha}$ and $n^{\beta}$.

Nevertheless, for the analysis of the experimental data we need measurable physical quantities that do not depend on the size of the reference phases.

The following procedure can be used for this purpose. At constant $T$ and $p$, the Gibbs-Duhem relationships for the two reference bulk phases are

$\sum_{i} x_{i}^{\alpha} \mathrm{d} \mu_{i}=0$

and

$\sum_{i} x_{i}^{\beta} \mathrm{d} \mu_{i}=0$. 
Using the above two relationships, it is possible to express $\mathrm{d} \mu_{1}$ and $\mathrm{d} \mu_{2}$ (i.e., the differential changes of the chemical potentials of two selected components) as a function of the other $\mathrm{d} \mu_{\mathrm{i}}$ values and the mole fractions at constant temperature and pressure:

$\mathrm{d} \mu_{1}=-\frac{x_{2}^{\alpha}}{x_{1}^{\alpha}} \mathrm{d} \mu_{2}-\sum_{i \neq 1,2} \frac{x_{i}^{\alpha}}{x_{1}^{\alpha}} \mathrm{d} \mu_{i}$.

and

$\mathrm{d} \mu_{2}=-\frac{x_{1}^{\beta}}{x_{2}^{\beta}} \mathrm{d} \mu_{1}-\sum_{i \neq 1,2} \frac{x_{i}^{\beta}}{x_{2}^{\beta}} \mathrm{d} \mu_{i}$.

Combining Eq. (35) with Eqs. (38) and (39), we obtain $-\mathrm{d} \gamma=\frac{1}{A} \sum_{i \neq 1,2}\left(n_{i}^{\sigma}+\frac{x_{2}^{\alpha} x_{i}^{\beta}-x_{2}^{\beta} x_{i}^{\alpha}}{x_{1}^{\alpha} x_{2}^{\beta}-x_{2}^{\alpha} x_{1}^{\beta}} n_{1}^{\sigma}+\frac{x_{1}^{\beta} x_{i}^{\alpha}-x_{1}^{\alpha} x_{i}^{\beta}}{x_{1}^{\alpha} x_{2}^{\beta}-x_{2}^{\alpha} x_{1}^{\beta}} n_{2}^{\sigma}\right) \mathrm{d} \mu_{i}$

or

$-\mathrm{d} \gamma=\sum_{i \neq 1,2}\left(\Gamma_{i}+\frac{x_{2}^{\alpha} x_{i}^{\beta}-x_{2}^{\beta} x_{i}^{\alpha}}{x_{1}^{\alpha} x_{2}^{\beta}-x_{2}^{\alpha} x_{1}^{\beta}} \Gamma_{1}+\frac{x_{1}^{\beta} x_{i}^{\alpha}-x_{1}^{\alpha} x_{i}^{\beta}}{x_{1}^{\alpha} x_{2}^{\beta}-x_{2}^{\alpha} x_{1}^{\beta}} \Gamma_{2}\right) \mathrm{d} \mu_{i}$.

By taking into account that

$\Gamma_{i}=\frac{1}{A}\left(n_{i}-n^{\alpha} x_{i}^{\alpha}-n^{\beta} x_{i}^{\beta}\right)$,

we have

$-\mathrm{d} \gamma=\frac{1}{A} \sum_{i \neq 1,2}\left(n_{i}+n_{1} \frac{x_{2}^{\alpha} x_{i}^{\beta}-x_{2}^{\beta} x_{i}^{\alpha}}{x_{1}^{\alpha} x_{2}^{\beta}-x_{2}^{\alpha} x_{1}^{\beta}}+n_{2} \frac{x_{1}^{\beta} x_{i}^{\alpha}-x_{1}^{\alpha} x_{i}^{\beta}}{x_{1}^{\alpha} x_{2}^{\beta}-x_{2}^{\alpha} x_{1}^{\beta}}\right) \mathrm{d} \mu_{i}$

or

$-\mathrm{d} \gamma=\sum_{i \neq 1,2}\left(\Gamma_{i}+\Gamma_{1} \frac{x_{2}^{\alpha} x_{i}^{\beta}-x_{2}^{\beta} x_{i}^{\alpha}}{x_{1}^{\alpha} x_{2}^{\beta}-x_{2}^{\alpha} x_{1}^{\beta}}+\Gamma_{2} \frac{x_{1}^{\beta} x_{i}^{\alpha}-x_{1}^{\alpha} x_{i}^{\beta}}{x_{1}^{\alpha} x_{2}^{\beta}-x_{2}^{\alpha} x_{1}^{\beta}}\right) \mathrm{d} \mu_{i}$.

Equation (44) can be written in the simpler form:

$-\mathrm{d} \gamma=\sum_{i \neq 1,2} \Gamma_{i}^{\prime} \mathrm{d} \mu_{i}$

where $\Gamma_{i}^{\prime}$ denotes the (relative) surface excess of component $i$ with respect to the two selected components, and

$\Gamma_{i}^{\prime}=\Gamma_{i}+\Gamma_{1} \frac{x_{2}^{\alpha} x_{i}^{\beta}-x_{2}^{\beta} x_{i}^{\alpha}}{x_{1}^{\alpha} x_{2}^{\beta}-x_{2}^{\alpha} x_{1}^{\beta}}+\Gamma_{2} \frac{x_{1}^{\beta} x_{i}^{\alpha}-x_{1}^{\alpha} x_{i}^{\beta}}{x_{1}^{\alpha} x_{2}^{\beta}-x_{2}^{\alpha} x_{1}^{\beta}}$.

It is clear that the $\Gamma_{i}^{\prime}$ values do not depend on the selection of the reference systems (that is, on the selection of $n^{\alpha}$ and $n^{\beta}$ ).
Therefore, the $\Gamma_{i}^{\prime}$ values can be determined as

$\Gamma_{i}^{\prime}=-\left(\frac{\partial \gamma}{\partial \mu_{i}}\right)_{T, p, \mu_{j \neq i}}=-\frac{1}{R T}\left(\frac{\partial \gamma}{\partial a_{i}}\right)_{T, p, a_{j \neq i}}$

(or more exactly $\Gamma_{i}^{\prime}=-\left(\frac{\partial \gamma}{\mathrm{d} \mu_{i(i \neq 1,2)}}\right)_{\mu_{i \neq t}}=-\frac{1}{R T}$ $\left(\frac{\partial \gamma}{\partial a_{i(i \neq 1,2)}}\right)_{T, p, a_{j \neq i}}$ ), where $a_{i}$ denotes the relative activity of component $i$.

Equation (45) (Gibbs adsorption isotherm also called the Gibbs adsorption equation) is one of the most important results from interfacial thermodynamics, and it is used all the time in physical chemistry and surface science.

In the following sections, we will briefly review the relevant mathematical background necessary for some of the derivations presented above.

\section{The mathematical background of interfacial thermodynamics}

\section{Homogeneous functions}

\section{Definition}

Let $f\left(x_{1}, x_{2}, \ldots, x_{m}\right)$ be a real function of variables $x_{1}, x_{2}$, $\ldots, x_{m}$. The function $f$ is a homogeneous function if for all values of the factor $k>0$

$f\left(k x_{1}, k x_{2}, \ldots, k x_{m}\right)=s(k) f\left(x_{1}, x_{2}, \ldots, x_{m}\right)$,

where the function $s(k)$ is usually called the scaling function, and is given by $s(k)=k^{n}$.

In other words, a homogeneous function is a function of one or several variables that satisfies the following condition: when all independent variables of a function are simultaneously multiplied by the same (arbitrary) factor, the value of the function is multiplied by some power of this factor. That is, if

$f\left(k x_{1}, k x_{2}, \ldots, k x_{m}\right)=k^{n} f\left(x_{1}, x_{2}, \ldots, x_{m}\right)$

for all $k>0$, then $f$ is said to be a homogeneous function of degree $n$. The degree $n$ can take on any value (positive, negative, or zero). A function $f$ is linearly homogenous if it is homogeneous of degree 1.

If for a function $f$ the equation

$f\left(k x_{1}, \ldots, k x_{m}, y_{1}, \ldots, y_{w}\right)=k^{n} f\left(x_{1}, \ldots, x_{m}, y_{1}, \ldots, y_{w}\right)$

is true, then we say that this function is homogeneous of degree $n$ in the variables $x_{1}, x_{2}, \ldots, x_{m}$. Such functions are called partly (or partially) homogeneous functions [1]. (It should be noted that it is possible for functions to be homogeneous of different degree in different variables, but 
here we restrict our attention to functions for which Eq. (M.2) or Eq. (M.3) holds).

Some remarks to the definition of homogeneous functions

Remark \#1 A homogeneous function of degree $n$ gives rise to a set of derivative functions that are homogeneous in the same set of variables and of degree $n-1$, that is, partial derivatives of a homogeneous function of degree $n$ are homogeneous functions of degree $n-1$.

Proof Differentiating both sides of Eq. (M.2) with respect to $x_{i}$ (for $i=1, \ldots, m$ ), we get

$k \frac{\partial f\left(k x_{1}, \ldots, k x_{m}\right)}{\partial k x_{i}}=k^{n} \frac{\partial f\left(x_{1}, \ldots, x_{m}\right)}{\partial x_{i}}$,

and then dividing both sides of Eq. (M.4) by $k$ we obtain

$\frac{\partial f\left(k x_{1}, \ldots, k x_{m}\right)}{\partial k x_{i}}=k^{n-1} \frac{\partial f\left(x_{1}, \ldots, x_{m}\right)}{\partial x_{i}}$.

Hence the derivatives of $f$ are homogeneous of degree $n-1$.

For example, in case of a homogeneous function of the first degree $f\left(k x_{1}, k x_{2}, \ldots, k x_{m}\right)=k f\left(x_{1}, x_{2}, \ldots, x_{m}\right)$ the partial derivative with respect to $x_{i}$ is $\frac{\partial f\left(k x_{1}, \ldots, k x_{m}\right)}{\partial k x_{i}} \frac{\partial k x_{i}}{\partial x_{i}}=$ $k \frac{\partial f\left(x_{1}, \ldots, x_{m}\right)}{\partial x_{i}}$, and therefore $\frac{\partial f\left(k x_{1}, \ldots, k x_{m}\right)}{\partial k x_{i}}=\frac{\partial f\left(x_{1}, \ldots, x_{m}\right)}{\partial x_{i}}$.

Remark \#2 Suppose that the domain of definition of the function $f$ lies in the first quadrant, $x_{1}>0, \ldots, x_{m}>0$, and contains the whole ray $\left(k x_{1}, \ldots, k x_{m}\right), k>0$, whenever it contains $\left(x_{1}, \ldots, x_{m}\right)$, i.e., it is assumed that for every point $\left(x_{1}, \ldots, x_{m}\right)$ in the domain of $f$, the point $\left(k x_{1}, \ldots,-\right.$ $k x_{m}$ ) also belongs to this domain for any $k>0$. Then $f$ is homogeneous of degree $n$ if and only if there exists a function $g$ of $m-1$ variables defined on the set of points of the form $\left(x_{2} / x_{1}, \ldots, x_{m} / x_{1}\right)$ such that for all $\left(x_{1}, \ldots, x_{m}\right)$ in the domain of definition [see for example refs. 32, 33]:

$f\left(x_{1}, \ldots, x_{m}\right)=x_{1}^{n} g\left(x_{2} / x_{1}, \ldots, x_{m} / x_{1}\right)$.

Proof Let $f\left(x_{1}, x_{2}, \ldots, x_{m}\right)$ be a homogeneous function of degree $n$ of variables $x_{1}, x_{2}, \ldots, x_{m}$ such that for all $k>0$

$f\left(k x_{1}, \ldots, k x_{m}\right)=k^{n} f\left(x_{1}, \ldots, x_{m}\right)$.

Obviously

$f\left(x_{1}, x_{2}, \ldots, x_{m}\right)=f\left(x_{1}, \frac{x_{2}}{x_{1}} x_{1}, \ldots, \frac{x_{m}}{x_{1}} x_{1}\right)$.

If we set $k=1 / x_{1}$, we have

$f\left(x_{1}, x_{2}, \ldots, x_{\mathrm{m}}\right)=x_{1}^{n} f\left(1, \frac{x_{2}}{x_{1}}, \ldots, \frac{x_{m}}{x_{1}}\right)$.
This means that $f$ is represented by

$f=x_{1}^{n} g\left(\frac{x_{2}}{x_{1}}, \ldots, \frac{x_{m}}{x_{1}}\right)$

with some function $g$. Since, conversely, every function $f$ formed by means of an appropriate function $g$ of $m-1$ variables satisfies the condition of homogeneity, the expression (M.10) represents the totality of homogeneous functions of degree $n$.

\section{Euler's theorem}

Euler's Theorem states that the differentiable function $f$ of $m$ variables is homogeneous of degree $n$, then the following identity holds:

$n f\left(x_{1}, x_{2}, \ldots, x_{m}\right)=\sum_{i=1}^{m} x_{i} \frac{\partial f}{\partial x_{i}}$.

Proof Let $f$ be a homogeneous function of degree $n$ such that

$f\left(\kappa \hat{x}_{1}, \kappa \hat{x}_{2}, \ldots, \kappa \hat{x}_{m}\right)=\kappa^{n} f\left(\hat{x}_{1}, \hat{x}_{2}, \ldots, \hat{x}_{m}\right)$,

and $k \neq 0, \quad \frac{1}{k}=\kappa, \quad x_{i}=\frac{1}{k} \hat{x}_{i}=\kappa \hat{x}_{i}, \quad k x_{i}=\hat{x}_{i}$.

Evidently

$$
\begin{aligned}
f\left(\kappa \hat{x}_{1}, \kappa \hat{x}_{2}, \ldots, \kappa \hat{x}_{m}\right) & =f\left(x_{1}, x_{2}, \ldots, x_{m}\right) \\
& =\left(\frac{1}{k}\right)^{n} f\left(k x_{1}, k x_{2}, \ldots, k x_{m}\right),
\end{aligned}
$$

and

$$
\begin{aligned}
k^{n} f\left(x_{1}, x_{2}, \ldots, x_{m}\right) & =f\left(k x_{1}, k x_{2}, \ldots, k x_{m}\right) \\
& =f\left(\hat{x}_{1}, \hat{x}_{2}, \ldots, \hat{x}_{m}\right),
\end{aligned}
$$

i.e., $f\left(x_{1}, x_{2}, \ldots, x_{m}\right)$ is also a homogeneous function of degree $n$.

Differentiating each side of formula (M.12) with respect to $\kappa$, we have the following relationship:

$$
\begin{array}{r}
\frac{\partial f\left(\kappa \hat{x}_{1}, \kappa \hat{x}_{2}, \ldots, \kappa \hat{x}_{m}\right)}{\partial \kappa \hat{x}_{1}} \hat{x}_{1}+\frac{\partial f\left(\kappa \hat{x}_{1}, \kappa \hat{x}_{2}, \ldots, \kappa \hat{x}_{m}\right)}{\partial \kappa \hat{x}_{2}} \hat{x}_{2}+\cdots \\
+\frac{\partial f\left(\kappa \hat{x}_{1}, \kappa \hat{x}_{2}, \ldots, \kappa \hat{x}_{m}\right)}{\partial \kappa \hat{x}_{m}} \hat{x}_{m}=n \kappa^{n-1} f\left(\hat{x}_{1}, \hat{x}_{2}, \ldots, \hat{x}_{m}\right) .
\end{array}
$$

Introducing $\kappa=\frac{1}{k}, \quad x_{i}=\kappa \hat{x}_{i}, \quad \hat{x}_{i}=k x_{i}$, Eq. (M.15) combined with Eq. (M.14) can be rewritten as

$$
\begin{array}{r}
\frac{\partial f\left(x_{1}, x_{2}, \ldots, x_{m}\right)}{\partial x_{1}} k x_{1}+\frac{\partial f\left(x_{1}, x_{2}, \ldots, x_{m}\right)}{\partial x_{2}} k x_{2}+\ldots \\
+\frac{\partial f\left(x_{1}, x_{2}, \ldots, x_{m}\right)}{\partial x_{m}} k x_{m}=n\left(\frac{1}{k}\right)^{n-1} k^{n} f\left(k x_{1}, k x_{2}, \ldots, k x_{m}\right) .
\end{array}
$$


This means that

$\sum_{i=1}^{m} \frac{\partial f\left(x_{1}, x_{2}, \ldots, x_{m}\right)}{\partial x_{i}} x_{i}=n f\left(x_{1}, x_{2}, \ldots, x_{m}\right)$,

or obviously

$\sum_{i=1}^{m} \frac{\partial f\left(\hat{x}_{1}, \hat{x}_{2}, \ldots, \hat{x}_{m}\right)}{\partial \hat{x}_{i}} \hat{x}_{i}=n f\left(\hat{x}_{1}, \hat{x}_{2}, \ldots, \hat{x}_{m}\right)$,

which is exactly Euler's theorem.

(Alternatively, by differentiating Eq. (M.2) with respect to $k$ we get

$\frac{\partial}{\partial k} f\left(k x_{1}, k x_{2}, \ldots, k x_{m}\right)=\frac{\partial}{\partial k} k^{n} f\left(x_{1}, x_{2}, \ldots, x_{m}\right)$

and thus

$\sum_{i=1}^{m} \frac{\partial f\left(k x_{1}, k x_{2}, \ldots, k x_{m}\right)}{\partial k x_{i}} x_{i}=n k^{n-1} f\left(x_{1}, x_{2}, \ldots, x_{m}\right)$.

Then, setting $k=1$

$\sum_{i=1}^{m} x_{i} \frac{\partial f\left(x_{1}, x_{2}, \ldots, x_{m}\right)}{\partial x_{i}}=n f\left(x_{1}, x_{2}, \ldots, x_{m}\right)$

which was to be proved).

We can show that the converse theorem also holds, that is if the function $f$ of the real variables $x_{1}, x_{2}, \ldots, x_{m}$ satisfies the identity (M.11), then the function $f$ is homogeneous of degree $n$.

Let us fix $\left(x_{1}, x_{2}, \ldots, x_{m}\right)$ and define the function $g$ of a single variable $t$ as

$g(t)=t^{-n} f\left(t x_{1}, \ldots, t x_{m}\right)-f\left(x_{1}, \ldots, x_{m}\right)$.

Differentiating each side of this equation with respect to $t$, we get

$$
\begin{aligned}
\frac{\mathrm{d} g(t)}{\mathrm{d} t}= & -n t^{-n-1} f\left(t x_{1}, \ldots, t x_{m}\right) \\
& +t^{-n} \sum_{i=1}^{m} x_{i} \frac{\mathrm{d} f\left(t x_{1}, \ldots, t x_{m}\right)}{\mathrm{d} t x_{i}} .
\end{aligned}
$$

By Euler's theorem, we have

$$
\sum_{i=1}^{m} \frac{\partial f\left(t x_{1}, \ldots, t x_{m}\right)}{\partial t x_{i}} t x_{i}=n f\left(t x_{1}, \ldots, t x_{m}\right) \text {, }
$$

so that

$$
\frac{\partial g(t)}{\partial t}=-n t^{-n-1} f\left(t x_{1}, \ldots, t x_{m}\right)+t^{-n} \frac{1}{t} n f\left(t x_{1}, \ldots, t x_{m}\right)
$$

Thus $g(t)$ is constant for all $t$. It is clear that $g(1)=0$, and therefore $g(t)=0$ for all $t$, and with Eq. (M.22) we get $t^{-n} f\left(t x_{1}, \ldots, t x_{m}\right)-f\left(x_{1}, \ldots, x_{m}\right)=0$,

and so

$f\left(t x_{1}, \ldots, t x_{m}\right)=t^{n} f\left(x_{1}, \ldots, x_{m}\right)$

for all $t>0$, which means that $f$ is homogeneous of degree $n$.

(Alternative proof: Let $g(t)=f\left(t x_{1}, \ldots, t x_{m}\right)$. After differentiation with respect to $t$ and by taking into account Eq. (M.11), we see that

$$
\begin{aligned}
\frac{\partial g(t)}{\partial t}= & x_{1} \frac{\partial f\left(t x_{1}, t x_{2}, \ldots, t x_{m}\right)}{\partial t x_{1}}+x_{2} \frac{\partial f\left(t x_{1}, t x_{2}, \ldots, t x_{m}\right)}{\partial t x_{2}} \\
& +\cdots+x_{m} \frac{\partial f\left(t x_{1}, t x_{2}, \ldots, t x_{m}\right)}{\partial t x_{m}} \\
= & \frac{1}{t}\left[t x_{1} \frac{\partial f\left(t x_{1}, t x_{2}, \ldots, t x_{m}\right)}{\partial t x_{1}}+t x_{2} \frac{\partial f\left(t x_{1}, t x_{2}, \ldots, t x_{m}\right)}{\partial t x_{2}} .\right. \\
& \left.+\cdots+t x_{m} \frac{\partial f\left(t x_{1}, t x_{2}, \ldots, t x_{m}\right)}{\partial t x_{m}}\right] \\
= & \frac{n}{t} f\left(t x_{1}, t x_{2}, \ldots, t x_{m}\right)=\frac{n}{t} g(t)
\end{aligned}
$$

This means that

$\frac{\mathrm{d} g(t)}{\mathrm{d} t}=\frac{n}{t} g(t)$.

After integration, we obtain for any $t>0$

$\ln |g(t)|=n \ln t+c$,

where $c$ is an integration constant, which is independent on $t$. This means that

$g(t)=e^{c} t^{n}$

Choosing $t=1$, we see that $g(1)=e^{c}$ and consequently $g(t)=g(1) t^{n}$, i.e., Eq. (M.27) holds).

\section{Some remarks to Euler's theorem}

Extensive variables in thermodynamics are those that depend linearly on the size of the system. This means that if a system is composed of several subsystems, the value of the extensive variable ("extensive quantity") for the composite system is calculated by summing over the subsystems. As a consequence, extensive thermodynamic functions are homogeneous functions of degree $n=1$ (homogeneous linear functions) with respect to their extensive arguments, i.e.,

$f\left(k x_{1}, \ldots, k x_{m}\right)=k f\left(x_{1}, \ldots, x_{m}\right)$,

and so

$\sum_{i=1}^{m} \frac{\partial f}{\partial x_{i}} x_{i}=f\left(x_{1}, \ldots, x_{m}\right)$. 
According to Eq. (M.5), partial derivatives of a homogeneous linear function are homogeneous functions of degree $n=0$ (homogeneous function of 0th degree), i.e.,

$f\left(k x_{1}, \ldots, k x_{m}\right)=f\left(x_{1}, \ldots, x_{m}\right)$

and

$\sum_{i=1}^{m} \frac{\partial f}{\partial x_{i}} x_{i}=0$.

Example \#1 Let us consider the following function:

$f(x, y, z)=-\frac{x^{3}}{y z}$.

Since

$f(k x, k y, k z)=-\frac{(k x)^{3}}{(k y)(k z)}=k\left(-\frac{x^{3}}{y z}\right)=k f(x, y, z)$,

the function $f(x, z, y)$ is homogeneous of the first degree in the variables $x, y$, and $z$. The partial derivatives are $\frac{\partial f}{\partial x}=-\frac{3 x^{2}}{y z}, \frac{\partial f}{\partial y}=\frac{x^{3}}{y^{2} z}$, and $\frac{\partial f}{\partial z}=\frac{x^{3}}{y z^{2}}$.

Thus, applying Euler's theorem

$$
\begin{aligned}
f(x, y, z) & =\frac{\partial f}{\partial x} \cdot x+\frac{\partial f}{\partial y} \cdot y+\frac{\partial f}{\partial z} \cdot z \\
& =\left(-\frac{3 x^{2}}{y z}\right) x+\left(\frac{x^{3}}{y^{2} z}\right) y+\left(\frac{x^{3}}{y z^{2}}\right) z=-\frac{x^{3}}{y z} .
\end{aligned}
$$

Example \#2 We know that partial derivatives of a homogeneous function of degree $n$ are homogeneous functions of degree $n-1$.

The partial derivative of the function $f$ (which is a homogeneous function of degree 1 , as defined by Eq. (E.1.1)) with respect to $x$ is

$f_{x}=\frac{\partial f}{\partial x}=-\frac{3 x^{2}}{y z}$.

The function $f_{x}$ is a homogeneous function of degree 0 in the variables $x, y$, and $z$, since

$f_{x}(k x, k y, k z)=-\frac{3(k x)^{2}}{(k y)(k z)}=-\frac{3 x^{2}}{y z}=f_{x}(x, y, z)$.

The partial derivatives are $\frac{\partial f_{x}}{\partial x}=-\frac{6 x}{y z}, \frac{\partial f_{x}}{\partial y}=\frac{3 x^{2}}{y^{2} z}$, and $\frac{\partial f_{x}}{\partial z}=\frac{3 x^{2}}{y z^{2}}$, that is

$\left(-\frac{6 x}{y z}\right) x+\left(\frac{3 x^{2}}{y^{2} z}\right) y+\left(\frac{3 x^{2}}{y z^{2}}\right) z=0$

in accordance with Euler's theorem.

\section{The Gibbs-Duhem Equation}

If the function $f\left(x_{1}, x_{2}, \ldots, x_{m}\right)$ is homogeneous of degree $n=1$ with respect to the variables $x_{1}, x_{2}, \ldots, x_{m}$, then one has the identity (M.2):

$f\left(k x_{1}, k x_{2}, \ldots, k x_{m}\right)=k f\left(x_{1}, x_{2}, \ldots, x_{m}\right)$.

Let us set $\frac{\partial f}{\partial x_{1}}=p_{1}, \ldots, \frac{\partial f}{\partial x_{m}}=p_{m}$, etc., and apply Euler's theorem to the function $f$.

We will obtain

$f=x_{1} p_{1}+x_{2} p_{2}+\cdots+x_{m} p_{m}$.

It results from this formula that the functions $p_{1}, p_{2}, \ldots, p_{m}$ are quantities (functions) of the same type as the quotient of energy or work by charge, mass, etc., and hence these are quantities (functions) of the same type as a potential, e.g., in thermodynamics, if $f$ is the internal energy function ( $U$, see "Models of the interfacial region" and "Adsorption" sections), and the $x_{i}$-s are the amounts of substances, we can call them partial molar internal energies of the constituents $1, \ldots, m$ in the system (thermodynamic or chemical potentials).

According to the considerations outlined above, the functions $p_{1}, p_{2}, \ldots, p_{\mathrm{m}}$ are homogeneous functions of degree zero in the variables $x_{1}, x_{2}, \ldots, x_{m}$. To each of these functions, we can apply Euler's theorem, and we will find the identities

$$
x_{1} \frac{\partial p_{1}}{\partial x_{1}}+x_{2} \frac{\partial p_{1}}{\partial x_{2}}+\cdots+x_{m} \frac{\partial p_{1}}{\partial x_{m}}=0
$$

$x_{1} \frac{\partial p_{m}}{\partial x_{1}}+x_{2} \frac{\partial p_{m}}{\partial x_{2}}+\cdots+x_{m} \frac{\partial p_{m}}{\partial x_{m}}=0$.

The identities

$\frac{\partial p_{i}}{\partial x_{j}}=\frac{\partial p_{j}}{\partial x_{i}}$,

which result from the definition of the functions (the mixed second partial derivatives are equal), permit the substitution of equations

$$
\begin{array}{r}
x_{1} \frac{\partial p_{1}}{\partial x_{1}}+x_{2} \frac{\partial p_{2}}{\partial x_{1}}+\cdots+x_{m} \frac{\partial p_{m}}{\partial x_{1}}=0 \\
\vdots \\
x_{1} \frac{\partial p_{1}}{\partial x_{m}}+x_{2} \frac{\partial p_{2}}{\partial x_{m}}+\cdots+x_{m} \frac{\partial p_{m}}{\partial x_{m}}=0,
\end{array}
$$

and therefore

$x_{1} \mathrm{~d} p_{1}+x_{2} \mathrm{~d} p_{2}+\cdots+x_{m} \mathrm{~d} p_{m}=0$.

This relation is known as Gibbs-Duhem equation or Gibbs-Duhem relation. 
Historical outlook Duhem was a great admirer of Gibbs, and extended a number of Gibbs' ideas. According to Miller [34] most probably Duhem was the first to use Euler's theorem explicitly to prove the Gibbs-Duhem equation. The first references appear in his book "Le Potentiel Thermodynamique" which was published in 1886 [35]. It is altogether fitting that his name be appended to the Gibbs-Duhem equation.

(An alternative derivation of the Gibbs-Duhem equation: consider an arbitrary function of

$x_{1}, x_{2}, \ldots, x_{m}: f\left(x_{1}, x_{2}, \ldots, x_{m}\right)$.

The total derivative (full derivative) of $f\left(x_{1}, x_{2}, \ldots, x_{m}\right)$ with respect to $x_{1}$ is

$$
\begin{aligned}
\frac{\mathrm{d} f}{\mathrm{~d} x_{1}} & =\frac{\partial f}{\partial x_{1}}+\frac{\partial f}{\partial x_{2}} \frac{\mathrm{d} x_{2}}{\mathrm{~d} x_{1}}+\cdots+\frac{\partial f}{\partial x_{m}} \frac{\mathrm{d} x_{m}}{\mathrm{~d} x_{1}} \\
& =p_{1}+p_{2} \frac{\mathrm{d} x_{2}}{\mathrm{~d} x_{1}}+\cdots+p_{m} \frac{\mathrm{d} x_{m}}{\mathrm{~d} x_{1}},
\end{aligned}
$$

where $p_{1}=\frac{\partial f}{\partial x_{1}}, p_{2}=\frac{\partial f}{\partial x_{2}}, p_{m}=\frac{\partial f}{\partial x_{m}}$, etc.

Multiplying both sides of the equation by the differential $\mathrm{d} x_{1}$ :

$$
\begin{aligned}
\mathrm{d} f & =\frac{\partial f}{\partial x_{1}} \mathrm{~d} x_{1}+\frac{\partial f}{\partial x_{2}} \mathrm{~d} x_{2}+\cdots+\frac{\partial f}{\partial x_{m}} \mathrm{~d} x_{m} \\
& =p_{1} \mathrm{~d} x_{1}+p_{2} \mathrm{~d} x_{2}+\cdots+p_{m} \mathrm{~d} x_{m} .
\end{aligned}
$$

The result will be the differential change $\mathrm{d} f$ in the function $f$. The differential of the form

$\mathrm{d} f=\sum_{i=1}^{m} p_{i}\left(x_{1}, x_{2}, \ldots, x_{m}\right) \mathrm{d} x_{i}$

is called the total differential or the exact differential of the function $f$.

According to Eq. (M.33)

$\sum_{i=1}^{m} p_{i} x_{i}=f\left(x_{1}, \ldots, x_{m}\right)$.

In order to get an expression for $\mathrm{d} f / \mathrm{d} x_{1}$ from (M.45) comparable with that in (M.42), we must differentiate (M.45) "generally," that is

$$
\begin{aligned}
\frac{\mathrm{d} f}{\mathrm{~d} x_{1}}= & p_{1}+x_{1} \frac{\partial p_{1}}{\partial x_{1}}+p_{2} \frac{\partial x_{2}}{\partial x_{1}}+x_{2} \frac{\partial p_{2}}{\partial x_{1}}+\cdots+p_{m} \frac{\partial x_{m}}{\partial x_{1}} \\
& +x_{m} \frac{\partial p_{m}}{\partial x_{1}} .
\end{aligned}
$$

There are thus two (general) expressions for $\mathrm{d} f / \mathrm{d} x_{1}$, both of which are correct. This can only be the case if

$x_{1} \frac{\partial p_{1}}{\partial x_{1}}+x_{2} \frac{\partial p_{2}}{\partial x_{1}}+\cdots+x_{m} \frac{\partial p_{m}}{\partial x_{1}}=0$ $x_{1} \mathrm{~d} p_{1}+x_{2} \mathrm{~d} p_{2}+\cdots+x_{m} \mathrm{~d} p_{m}=\sum_{i}^{m} x_{i} \mathrm{~d} p_{i}=0$

which is the Gibbs-Duhem equation).

Example \#3 Consider the function

$f(x, y, z)=-\frac{x^{3}}{y z}$

which is homogeneous of degree 1 (see Example \#1).

The partial derivatives with respect to $x, y$, and $z$ are

$f_{x}^{\prime}=\frac{\partial f}{\partial x}=-\frac{3 x^{2}}{y z}, \quad f_{y}^{\prime}=\frac{\partial f}{\partial y}=\frac{x^{3}}{y^{2} z}$, and $f_{z}^{\prime}=\frac{\partial f}{\partial z}=\frac{x^{3}}{y z^{2}}$.

The partial derivatives of $f_{x}, f_{y}$, and $f_{z}$ with respect to $x$ can be given as

$\frac{\partial f_{x}^{\prime}}{\partial x}=-\frac{6 x}{y z}, \quad \frac{\partial f_{y}^{\prime}}{\partial x}=\frac{3 x^{2}}{y^{2} z}$ and $\frac{\partial f_{z}^{\prime}}{\partial x}=\frac{3 x^{2}}{y z^{2}}$

In accordance with Eq. (M.39)

$x \frac{-6 x}{y z}+y \frac{3 x^{2}}{y^{2} z}+z \frac{3 x^{2}}{y z^{2}}=\frac{-6 x^{2}+3 x^{2}+3 x^{2}}{y z}=0$.

Alternatively, we can formally write

$\mathrm{d} f_{x}^{\prime}=\frac{-6 x y z \cdot \mathrm{d} x+3 x^{2} z \cdot \mathrm{d} y+3 x^{2} y \cdot \mathrm{d} z}{y^{2} z^{2}}$

$\mathrm{d} f_{y}^{\prime}=\frac{3 x^{2} y^{2} z \cdot \mathrm{d} x-2 x^{3} y z \cdot \mathrm{d} y-x^{3} y^{2} \cdot \mathrm{d} z}{y^{4} z^{2}}$

and

$\mathrm{d} f_{z}^{\prime}=\frac{3 x^{2} y z^{2} \cdot \mathrm{d} x-x^{3} z^{2} \cdot \mathrm{d} y-2 x^{3} y z \cdot \mathrm{d} z}{y^{2} z^{4}}$.

Thus,

$$
\begin{aligned}
x \mathrm{~d} f_{x}^{\prime}+y \mathrm{~d} f_{y}^{\prime}+z \mathrm{~d} f_{z}^{\prime}= & \frac{-6 x^{2} y^{2} z^{2} \cdot \mathrm{d} x+3 x^{3} y z^{2} \cdot \mathrm{d} y+3 x^{3} y^{2} z \cdot \mathrm{d} z}{z^{3} y^{3}} \\
& +\frac{3 x^{2} y^{2} z^{2} \cdot \mathrm{d} x-2 x^{3} y z^{2} \cdot \mathrm{d} y-x^{3} y^{2} z \cdot \mathrm{d} z}{z^{3} y^{3}} \\
& +\frac{3 x^{2} y^{2} z^{2} \cdot \mathrm{d} x-x^{3} y z^{2} \cdot \mathrm{d} y-2 x^{3} y^{2} z \cdot \mathrm{d} z}{z^{3} y^{3}}=0
\end{aligned}
$$

in accordance with the Gibbs-Duhem equation (M.40).

Example \#4 Consider the internal energy function $U$ defined by

$U=U\left(S, V, n_{1}, \ldots, n_{m}\right)$

or 
where $S$ is the entropy, $V$ is the volume, and $n_{i}(i=1, \ldots, m)$ is the chemical amount of component $i$. Since $U$ is a homogeneous function of degree 1 with respect to all of its variables

$$
U\left(k S, k V, k n_{1}, \ldots, k n_{m}\right)=k U\left(S, V, n_{1}, \ldots, n_{m}\right),
$$

The temperature $(T)$, pressure $(p)$, and the chemical potentials $\mu_{i} \quad(i=1, \ldots, m)$ of the components are $T=\left(\frac{\partial U}{\partial S}\right)_{V, n_{1}, \ldots, n_{m}}, p=-\left(\frac{\partial U}{\partial V}\right)_{S, n_{1}, \ldots, n_{m}}, \quad \mu_{i}=\left(\frac{\partial U}{\partial n_{i}}\right)_{S, V, n_{i \neq m}}$ respectively. (In the above equations, $n_{i}$ denotes the set $\left[n_{1}, \ldots, n_{m}\right](i=1, \ldots, m)$ and $n_{j \neq i}$ denotes all the elements (variables) in $\left[n_{1}, \ldots, n_{m}\right]$ except for the $i$ th.) According to Euler's theorem

$U=T S+(-p V)+\sum_{i} \mu_{i} n_{i}$.

The Gibbs-Duhem relation is written as

$-S \mathrm{~d} T+V \mathrm{~d} p+n_{1} \mathrm{~d} \mu_{1}+\cdots+n_{m} \mathrm{~d} \mu_{m}=0$.

It follows that $T, p$, and $\mu_{i}$ cannot be independently variable, i.e., the intensive variables are not independent. If we know $m-1$ of them, the value of the $m$ th can be determined from the Gibbs-Duhem equation. It is particularly useful in its application to changes at constant temperature and pressure, and it may be written as

$\sum_{i=1}^{m} n_{i} \mathrm{~d} \mu_{i}=0$.

\section{Partly homogeneous functions of degree 1}

A function $f$ is called "partly homogeneous" of degree 1 in terms of $m$ among $m+w$ variables if

$$
\begin{aligned}
& f\left(k x_{1}, \ldots, k x_{m}, y_{1}, \ldots, y_{w}\right)=k f\left(x_{1}, \ldots, x_{m}, y_{1}, \ldots, y_{w}\right) \\
& \quad(k>0),
\end{aligned}
$$

i.e., the function $f$ is homogeneous with respect to certain variables $\left(x_{1}, x_{2}, \ldots, x_{m}\right)$, but not homogeneous with respect to all of the variables. These functions are important as they are frequently encountered in thermodynamics [36].

Let us introduce new variables

$z_{i}=x_{1} \cdot y_{i}$

and the function $\hat{f}$ as

$\hat{f}\left(x_{1}, \ldots, x_{m}, z_{1}, \ldots, z_{w}\right)=f\left(x_{1}, \ldots, x_{m}, \frac{z_{1}}{x_{1}}, \ldots, \frac{z_{w}}{x_{1}}\right)$.

We can prove that $\hat{f}$ is homogeneous of the first order with respect to all of its variables. According to Eq. (M.51)

$$
\begin{aligned}
\hat{f}\left(k x_{1}, \ldots, k x_{m}, k z_{1}, \ldots, k z_{w}\right) & =f\left(k x_{1}, \ldots k x_{m}, \frac{k z_{1}}{k x_{1}}, \ldots, \frac{k z_{w}}{k x_{1}}\right) \\
& =f\left(k x_{1}, \ldots k x_{m}, \frac{z_{1}}{x_{1}}, \ldots, \frac{z_{w}}{x_{1}}\right)
\end{aligned}
$$

On the other hand, $f$ is a homogeneous linear function with respect to $\left(x_{1}, x_{2}, \ldots, x_{m}\right)$. Thus

$f\left(k x_{1}, \ldots, k x_{m}, \frac{z_{1}}{x_{1}}, \ldots, \frac{z_{w}}{x_{1}}\right)=k f\left(x_{1}, \ldots, x_{m}, \frac{z_{1}}{x_{1}}, \ldots, \frac{z_{w}}{x_{1}}\right)$.

By taking into account the definition of $\hat{f}$,

$\hat{f}\left(k x_{1}, \ldots, k x_{m}, k z_{1}, \ldots, k z_{w}\right)=k \hat{f}\left(x_{1}, \ldots, x_{m}, z_{1}, \ldots, z_{w}\right)$

which was to be proved.

The partial derivatives of $f$ and $\hat{f}$ with respect to $x_{i}, y_{i}$, and $z_{i}$ are, respectively, given by

$$
\begin{aligned}
f_{i}^{x} & =\left(\frac{\partial f}{\partial x_{i}}\right)_{x_{j \neq i}, y_{j}}, \quad f_{i}^{y}=\left(\frac{\partial f}{\partial y_{i}}\right)_{x_{j}, y_{j \neq i}}, \quad \hat{f}_{i}^{x}=\left(\frac{\partial \hat{f}}{\partial x_{i}}\right)_{x_{j \neq i}, z_{j}}, \\
\hat{f}_{i}^{z} & =\left(\frac{\partial \hat{f}}{\partial z_{i}}\right)_{x_{j}, z_{j \neq i}}
\end{aligned}
$$

For $i=1, \ldots, m$, the partial derivatives of $\hat{f}$ are given as $\hat{f}_{i}^{x}=\frac{\partial \hat{f}}{\partial x_{i}}=\frac{\partial f}{\partial x_{i}}=f_{i}^{x}$

and for $j=1, \ldots, w$

$\hat{f}_{j}^{z}=\frac{\partial \hat{f}}{\partial z_{j}}=\frac{\partial f}{\partial y_{j}} \cdot \frac{1}{x_{1}}=f_{j}^{y} \cdot \frac{1}{x_{1}}$.

Thus, the total derivative of $\hat{f}$ is

$$
\begin{aligned}
\frac{\partial \hat{f}}{\partial x_{1}}= & \frac{\partial f}{\partial x_{1}}+\frac{\partial f}{\partial y_{1}} \cdot\left(\frac{-z_{1}}{x_{1}^{2}}\right)+\frac{\partial f}{\partial y_{2}} \cdot\left(\frac{-z_{2}}{x_{1}^{2}}\right)+\cdots+\frac{\partial f}{\partial y_{w}} \\
& \cdot\left(\frac{-z_{w}}{x_{1}^{2}}\right) .
\end{aligned}
$$

Using Eqs. (M.50) and (M.55, M.56), Eq. (M.58) can be rewritten in the form

$\hat{f}_{1}^{x}=f_{1}^{x}+f_{1}^{y} \cdot\left(\frac{-y_{1}}{x_{1}}\right)+f_{2}^{y} \cdot\left(\frac{-y_{2}}{x_{1}}\right)+\cdots+f_{w}^{y} \cdot\left(\frac{-y_{w}}{x_{1}}\right)$

and with Eq. (M.57)

$\hat{f}_{1}^{x}=f_{1}^{x}-\sum_{i=1}^{w} \hat{f}_{i}^{z} y_{i}$ 
Since $\hat{f}$ is homogeneous of the first degree with respect to all of its variables, and its partial derivatives are homogeneous of degree zero, we can apply Euler's theorem. Hence

$\hat{f}=\sum_{i=1}^{m} x_{i} \hat{f}_{i}^{x}+\sum_{j=1}^{w} z_{j} \hat{f}_{j}^{z}$

and

$0=\sum_{i=1}^{m} x_{i} \mathrm{~d} \hat{f}_{i}^{x}+\sum_{j=1}^{w} z_{j} \mathrm{~d} \hat{f}_{j}^{z}$

With Eqs. (M.51) and (M.56-M.62), we have

$f=x_{1}\left(f_{1}^{x}-\sum_{j=1}^{w} \frac{f_{j}^{y}}{x_{1}} y_{j}\right)+\sum_{i=2}^{m} x_{i} f_{i}^{x}+\sum_{j=1}^{w} x_{1} y_{j} \frac{f_{j}^{y}}{x_{1}}$,

and

$0=x_{1} \mathrm{~d}\left(f_{1}^{x}-\sum_{j=1}^{w} \frac{f_{j}^{y}}{x_{1}} y_{j}\right)+\sum_{i=2}^{m} x_{i} \mathrm{~d} f_{i}^{x}+\sum_{j=1}^{w} x_{1} y_{j} \mathrm{~d} \frac{f_{j}^{y}}{x_{1}}$.

Consequently

$f=\sum_{i=1}^{m} x_{i} f_{i}^{x}=\sum_{i=1}^{m} x_{i}\left(\frac{\partial f}{\partial x_{i}}\right)_{x_{j \neq i}, y_{j}}$

and

$0=\sum_{i=1}^{m} x_{i} \mathrm{~d} f_{i}^{x}-\sum_{j=1}^{w} f_{i}^{y} \mathrm{~d} y_{i}$.

The latter relation is the Gibbs-Duhem equation for "partly homogeneous functions."

Example \#5 The function

$f=-\frac{x^{2}}{y} w+u^{5} x$

is not fully homogeneous, since

$f(k x, k y, k u, k w) \neq k^{n} f(x, y, u, w)$.

(If the powers of $x, y, u$, and $w$ are added, the first term on the right-hand side of the expression yields 2 , and the second term 6.)

However, if $u$ and $w$ are constant, the sum of the powers of $x$ and $y$ for each term is 1 . Therefore, the function is partly homogeneous (with respect to $x$ and $y$ ), so that

$f(k x, k y, u, w)=k f(x, y, u, w)$.

The partial derivatives with respect to $x$ and $y$ are

$f_{x}^{\prime}=\frac{\partial f}{\partial x}=-\frac{2 x w}{y}+u^{5}$ and

$f_{y}^{\prime}=\frac{\partial f}{\partial y}=\frac{x^{2} w}{y^{2}}$

Thus, applying Euler's theorem

$f(x, y, u, w)=\left(-\frac{2 x w}{y}+u^{5}\right) x+\left(\frac{x^{2} w}{y^{2}}\right) y=-\frac{x^{2} w}{y}+u^{5} x$.

The partial derivatives with respect to $u$ and $w$ are

$f_{u}^{\prime}=\frac{\partial f}{\partial u}=5 u^{4} x$

and

$f_{w}^{\prime}=\frac{\partial f}{\partial w}=-\frac{x^{2}}{y}$

We can formally write

$x \cdot \mathrm{d} f_{x}^{\prime}=\frac{-2 w y x \cdot \mathrm{d} x-2 x^{2} y \cdot \mathrm{d} w+2 x^{2} w \cdot \mathrm{d} y}{y^{2}}+5 u^{4} x \cdot \mathrm{d} u$,

and

$y \cdot \mathrm{d} f_{y}^{\prime}=\frac{2 x w y^{2} \cdot \mathrm{d} x+x^{2} y^{2} \cdot \mathrm{d} w-2 x^{2} w y \cdot \mathrm{d} y}{y^{3}}$.

Similarly

$f_{u}^{\prime} \cdot \mathrm{d} u=5 u^{4} x \cdot \mathrm{d} u$,

$f_{w}^{\prime} \cdot \mathrm{d} w=-\frac{x^{2}}{y} \cdot \mathrm{d} w$

Thus,

$$
\begin{aligned}
x \mathrm{~d} f_{x}^{\prime}+y \mathrm{~d} f_{y}^{\prime}-f_{u}^{\prime} \mathrm{d} u-f_{w}^{\prime} \mathrm{d} w \\
=\frac{-2 w y x \cdot \mathrm{d} x-2 x^{2} y \cdot \mathrm{d} w+2 x^{2} w \cdot \mathrm{d} y}{y^{2}}+5 u^{4} x \cdot \mathrm{d} u \\
\quad+\frac{2 x w y^{2} \cdot \mathrm{d} x+x^{2} y^{2} \cdot \mathrm{d} w-2 x^{2} w y \cdot \mathrm{d} y}{y^{3}} \\
\quad-5 u^{4} x \cdot \mathrm{d} u-\left(-\frac{x^{2}}{y} \cdot \mathrm{d} w\right)=0
\end{aligned}
$$

in accordance with the Gibbs-Duhem equation for "partly homogeneous functions" (Eq. M.66).

Example \#6 The function

$f=-\frac{x^{2}}{y} w+u^{3} x$

is not fully homogeneous, since $f(k x, k y, k u, k w) \neq$ $k^{n} f(x, y, u, w)$. 
Let us introduce new variables $\alpha=x \cdot u$ and $\beta=x \cdot w$, i.e., $u=\alpha / x$ and $w=\beta / x$, and the function $\hat{f}$ as

$\hat{f}=-\frac{x^{2}}{y} \frac{\beta}{x}+\left(\frac{\alpha}{x}\right)^{3} x=-\frac{x \beta}{y}+\frac{\alpha^{3}}{x^{2}}$.

Since

$$
\begin{aligned}
\hat{f}(k x, k y, k \alpha, k \beta) & =-\frac{k x \cdot k \beta}{k y}+\frac{(k \alpha)^{3}}{(k x)^{2}}=k\left(-\frac{x \cdot \beta}{y}+\frac{\alpha^{3}}{x^{2}}\right) \\
& =k \hat{f}(x, y, \alpha, \beta),
\end{aligned}
$$

it is obvious that $\hat{f}$ is a homogeneous function of degree 1 with respect to all of its variables $(x, y, \alpha, \beta)$.

Example \#7 Let us consider the Gibbs free energy function $G$ defined by

$G=G\left(T, p, n_{1}, n_{2}, \ldots, n_{m}\right)$,

where $T$ is the temperature, $p$ is the pressure, and $n_{i}$ $(i=1, \ldots, m)$ is the chemical amount of component $i$. At given (constant) $T$ and $p$, the function $G$ is partly homogeneous of degree one in terms of the variables $n_{1}, \ldots, n_{m}$. We thus have

$G\left(T, p, k n_{1}, \ldots, k n_{m}\right)=k G\left(T, p, n_{1}, \ldots, n_{m}\right)$.

The total differential of $G$ is given as

$\mathrm{d} G=\left(\frac{\partial G}{\partial T}\right)_{p, n_{i}} \mathrm{~d} T+\left(\frac{\partial G}{\partial p}\right)_{T, n_{i}} \mathrm{~d} p+\sum_{i=1}^{m}\left(\frac{\partial G}{\partial n_{i}}\right)_{T, p, n_{j \neq i}} \mathrm{~d} n_{i}$.

The entropy $(S)$, volume $(V)$, and the chemical potentials $\mu_{i} \quad(i=1, \ldots, m)$ of the components are $S=-\left(\frac{\partial G}{\partial T}\right)_{p, n_{i}}$, $V=\left(\frac{\partial G}{\partial p}\right)_{T, n_{i}}$, and $\mu_{i}=\left(\frac{\partial G}{\partial n_{i}}\right)_{T, p, n_{j \neq i}}$, respectively. Thus, Eq. (E.7.3) can be rewritten as

$\mathrm{d} G=-S \mathrm{~d} T+V \mathrm{~d} p+\sum_{i=1}^{m} \mu_{i} \mathrm{~d} n_{i}$

According to Euler's theorem

$G=\sum_{i} \mu_{i} n_{i}$

In order to obtain an expression for $\mathrm{d} G$ from (E.7.5) comparable with that in (E.7.3), we must differentiate (E.7.5). This gives

$\mathrm{d} G=\sum_{i=1}^{m} \mu_{i} \mathrm{~d} n_{i}+\sum_{i=1}^{m} n_{i} \mathrm{~d} \mu_{i}$

and by comparing Eq. (E.7.6) with Eq. (E.7.4) we obtain the following important relation (i.e., the Gibbs-Duhem equation):
$S \mathrm{~d} T-V \mathrm{~d} p+\sum_{i} n_{i} \mathrm{~d} \mu_{i}=0$.

\section{Legendre transformation}

(The transform is named after the French mathematician Adrien-Marie Legendre (1752-1833).)

Let $f\left(x_{1}, x_{2}, \ldots, x_{m}\right)$ be an arbitrary analytic function of variables $x_{1}, x_{2}, \ldots, x_{m}$. The differential of $f$ is

$\begin{aligned} \mathrm{d} f & =\frac{\partial f}{\partial x_{1}} \mathrm{~d} x_{1}+\frac{\partial f}{\partial x_{2}} \mathrm{~d} x_{2}+\cdots+\frac{\partial f}{\partial x_{m}} \mathrm{~d} x_{m} \\ & =p_{1} \mathrm{~d} x_{1}+p_{2} \mathrm{~d} x_{2}+\cdots+p_{m} \mathrm{~d} x_{m} .\end{aligned}$

Consider a new function $g$ of the variables $p_{1}$ and $x_{2}$, $x_{3}, \ldots, x_{m}$ :

$g\left(p_{1}, x_{2}, \ldots, x_{m}\right)=f\left(x_{1}\left(p_{1}\right), x_{2}, \ldots, x_{m}\right)-p_{1} x_{1}\left(p_{1}\right)$,

where $p_{1}=\frac{\partial f}{\partial x_{1}}$. A necessary condition is the existence of a one-to-one relation between $p_{1}$ and $x_{1}$, that is, the function $p_{1}\left(x_{1}, x_{2}, \ldots, x_{m}\right)$ can be inverted to give $x_{1}\left(p_{1}\right)$ (This means that $p_{1}$ is bijective).

The new function $g\left(p_{1}, x_{2}, \ldots, x_{m}\right)$ is called the Legendre transform of the function $f\left(x_{1}, x_{2}, \ldots, x_{m}\right)$. A Legendre transform converts from a function of one set of variables to another function of a "conjugate" set of variables. In general, this is a special transformation that allows us to replace variables in a function in a consistent manner.

The differential of $g\left(p_{1}, x_{2}, \ldots, x_{m}\right)$ is

$\mathrm{d} g=\mathrm{d} f-x_{1}\left(p_{1}\right) \mathrm{d} p_{1}-p_{1}\left(x_{1}, x_{2}, \ldots, x_{m}\right) \mathrm{d} x_{1}$.

With Eq. (M.67)

$\mathrm{d} g=-x_{1}\left(p_{1}\right) \mathrm{d} p_{1}+\frac{\partial f}{\partial x_{2}} \mathrm{~d} x_{2}+\cdots+\frac{\partial f}{\partial x_{m}} \mathrm{~d} x_{m}$.

Formally, the total differential of $g$ is

$\mathrm{d} g=\frac{\partial g}{\partial p_{1}} \mathrm{~d} p_{1}+\frac{\partial g}{\partial x_{2}} \mathrm{~d} x_{2}+\cdots+\frac{\partial g}{\partial x_{m}} \mathrm{~d} x_{m}$.

Comparing Eqs. (M.70) and (M.71) $x_{1}=-\frac{\partial g}{\partial p_{1}}, \frac{\partial f}{\partial x_{2}}=\frac{\partial g}{\partial x_{2}}$, $\frac{\partial f}{\partial x_{3}}=\frac{\partial g}{\partial x_{3}}$, etc. Let us take a look at some simple examples to see how this works.

Example \#8 One-dimensional Legendre transformation

Consider an arbitrary function of $x: f(x)$. We know that locally the slope of this curve is precisely its derivative with respect to $x$, so the change in the function $f(x)$ at the point $x$ for a small change in the argument $\mathrm{d} x$ is

$\mathrm{d} f=\frac{\partial f}{\partial x} \mathrm{~d} x \equiv p(x) \mathrm{d} x$

where $p=\frac{\partial f(x)}{\partial x}=f^{\prime}(x)$, as usual. Now suppose that we want to find a function that reverses the roles of the slope and infinitesimal, i.e., a function $g(p)$ such that 
$\mathrm{d} g=x \mathrm{~d} p$ (where we now view $x$ as a function of $p$ defined by the inverse of $p=f^{\prime}(x)$ ). We can see that the function

$g=f(p)-p x(p)$

(the Legendre transform) has the desired property:

$\mathrm{d} g=\mathrm{d} f-x \mathrm{~d} p-p \mathrm{~d} x=-x \mathrm{~d} p$.

Notice that since $g(p)$ is a function of $p$ only, we must have

$\frac{\mathrm{d} g(p)}{\mathrm{d} p}=-x$.

Consequently, we have a pair of functions $f(x)$ and $g(p)$ related in the following way:

$f(x) \rightarrow g(p)=f(x(p))-x(p) p \quad \ldots \quad x(p): \frac{\mathrm{d} f(x)}{\mathrm{d} x}=p$,

$g(p) \rightarrow f(x)=g(p(x))-p(x) x \quad \ldots \quad p(x): \frac{\mathrm{d} g(p)}{\mathrm{d} p}=-x$,

where $f(x)$ and $g(p)$ are Legendre transforms of each other. There is a certain symmetry here, and the same transformation takes us back and forth.

Consider the function $f(x)=a x^{2}$. In this case $p=\frac{\mathrm{d} f(x)}{\mathrm{d} x}=2 a x$, and $x=\frac{p}{2 a}$. The Legendre transform of $f$ is

$$
\begin{aligned}
L[f(x)] & =f(x)-p \cdot x=a x^{2}-2 a x^{2}=\frac{p^{2}}{4 a}-\frac{p^{2}}{2 a}=-\frac{p^{2}}{4 a} \\
& =g(p) .
\end{aligned}
$$

On the other hand, $g(p)=-\frac{p^{2}}{4 a}, \frac{\mathrm{d} g(p)}{\mathrm{d} p}=-\frac{p}{2 a}=-x$, and the Legendre transform of $g(p)$ is given as

$$
\begin{aligned}
L[g(p)] & =g(p)-p \cdot(-x)=-\frac{p^{2}}{4 a}+\frac{p^{2}}{2 a}=\frac{p^{2}}{4 a}=a x^{2} \\
& =f(x) .
\end{aligned}
$$

Remark To avoid the minus sign in Eq. (E.8.4), the Legendre transform can alternatively be defined as

$$
g=p x-f
$$

(for functions of several variables: $g\left(p_{1}, x_{2}, \ldots, x_{m}\right)=p_{1-}$ $\left.x_{1}\left(p_{1}\right)-f\left(x_{1}\left(p_{1}\right), x_{2}, \ldots, x_{m}\right)\right)$, in which case the Legendre transform is its own inverse, since $f=p x-g$, rather than being the negative of its inverse. This opposite-sign alternative definition has the advantage that it gives rise to the symmetric identity $f+g=p x$ which in words says that the sum of a function and its Legendre transform equals the product of the conjugate pair of variables. It is worth to emphasize the dimensional consistency of this identity. $f+g$ is actually a function of either $p$ or $x$ but not both, because one variable implicitly depends on the other via a Legendre transform.

Usually, the definition given in Eq. (M.68) is preferred in thermodynamics.

Example \#9 Consider the function

$f(x, z)=x^{2}+y^{2}$.

The partial derivatives are $\frac{\partial f}{\partial x}=2 x=p_{1}, \frac{\partial f}{\partial y}=2 y=p_{2}$, $x=\frac{p_{1}}{2}$, and $y=\frac{p_{2}}{2}$.

The Legendre transforms of $f$ are

$g\left(x, p_{2}\right)=x^{2}+y^{2}-p_{2} \cdot y=x^{2}+\frac{p_{2}^{2}}{4}-\frac{p_{2}^{2}}{2}=x^{2}-\frac{p_{2}^{2}}{4}$,

$g\left(p_{1}, y\right)=x^{2}+y^{2}-p_{1} \cdot x=\frac{p_{1}^{2}}{4}+y^{2}-\frac{p_{1}^{2}}{2}=y^{2}-\frac{p_{1}^{2}}{4}$,

$$
\begin{aligned}
g\left(p_{1}, p_{2}\right) & =x^{2}+y^{2}-p_{1} \cdot x-p_{2} \cdot y=\frac{p_{1}^{2}}{4}+\frac{p_{2}^{2}}{4}-\frac{p_{1}^{2}}{2}-\frac{p_{2}^{2}}{2} \\
& =-\frac{1}{4}\left(p_{1}^{2}+p_{2}^{2}\right) .
\end{aligned}
$$

Example \#10 Consider the function

$f(x, y, z)=-\frac{x^{3}}{y z}$.

The partial derivatives are $\frac{\partial f}{\partial x}=-\frac{3 x^{2}}{y z}=f_{x}, \frac{\partial f}{\partial y}=\frac{x^{3}}{y^{2} z}=f_{y}$, and $\frac{\partial f}{\partial z}=\frac{x^{3}}{y z^{2}}=f_{z}$.

The variable $y$ can be expressed as $y=\frac{x^{3 / 2}}{z^{1 / 2} f_{y}^{1 / 2}}$.

Define the new function $f_{2}\left(x, f_{y}, z\right)$ as follows:

$$
\begin{aligned}
f_{2}\left(x, f_{y}, z\right)=-\frac{x^{3}}{y z}-y f_{y} & =-\frac{x^{3} z^{1 / 2} f_{y}^{1 / 2}}{z x^{3 / 2}}-\frac{x^{3 / 2}}{z^{1 / 2} f_{y}^{1 / 2}} f_{y} \\
& =-\frac{2 x^{3 / 2} f_{y}^{1 / 2}}{z^{1 / 2}} .
\end{aligned}
$$

It is obvious from this definition that $f_{2}$ is the Legendre transform of $f$ with respect to $y$. Taking into account that

$\frac{\partial f_{2}}{\partial z}=-2 x^{3 / 2} f_{y}^{1 / 2}\left(-\frac{1}{2} z^{-3 / 2}\right)=\frac{x^{3 / 2} f_{y}^{1 / 2}}{z^{3 / 2}}=f_{z}$,

and

$z=\frac{x f_{y}^{1 / 3}}{f_{z}^{2 / 3}}$,

the Legendre transformation of $f_{2}$ (with respect to $z$ ) yields 


$$
\begin{aligned}
f_{3}\left(x, f_{y}, f_{z}\right) & =f_{2}\left(x, f_{y}, z\right)-z f_{z} \\
& =-\frac{2 x^{3 / 2} f_{y}^{1 / 2}}{x^{1 / 2} f_{y}^{1 / 6}} f_{z}^{1 / 3}-\frac{x f_{y}^{1 / 3}}{f_{z}^{2 / 3}} f_{z}=-3 x f_{y}^{1 / 3} f_{z}^{1 / 3} .
\end{aligned}
$$

Alternatively, Eq. (E.10.5) can be derived directly by performing two successive Legendre transformations

$f_{3}\left(x, f_{y}, f_{z}\right)=f(x, y, z)-y f_{y}-z f_{z}$

with $f(x, y, z)=-\frac{x^{3}}{y z}, \quad \frac{\partial f}{\partial y}=\frac{x^{3}}{y^{2} z}=f_{y}, \quad \frac{\partial f}{\partial z}=\frac{x^{3}}{y z^{2}}=f_{z}, \quad y=$ $x f_{y}^{-2 / 3} f_{z}^{1 / 3}$, and $z=x f_{y}^{1 / 3} f_{z}^{-2 /-3}$.

Thus

$$
\begin{aligned}
f(x, y, z)-y f_{y}-z f_{z}= & \frac{-x}{x f_{y}^{-2 / 3} f_{z}^{1 / 3} \cdot x f_{y}^{1 / 3} f_{z}^{-2 / 3}}-x f_{y}^{-2 / 3} f_{z}^{1 / 3} \\
& \cdot f_{y}-x f_{y}^{1 / 3} f_{z}^{-2 / 3} \cdot f_{z} \\
= & -3 x f_{y}^{1 / 3} f_{z}^{1 / 3} .
\end{aligned}
$$

We remark that while $f$ is a homogeneous function of the first degree in the variables $x, y$, and $z$ (see Example \#1), the Legendre transforms $f_{2}\left(x, f_{y}, z\right)$ and $f_{3}\left(x, f_{y}, f_{z}\right)$ are only partly homogeneous (the first with respect to $x$ and $z$, and the second with respect to only $x$ ).

Example \#11 Consider the function

$f(x, y, u, w)=-\frac{x^{2} w}{y}+u^{5} x$.

Since $f_{y}=\frac{\partial f}{\partial y}=\frac{x^{2} w}{y^{2}}$, the Legendre transformation of $f$ with respect to $y$ yields

$L_{y}[f(x, y, u, w)]=f_{2}\left(x, f_{y}, u, w\right)=-2 x w^{1 / 2} f_{y}^{1 / 2}+u^{5} x$.

Note that $f(x, y, u, w)$ is a partly homogeneous function of degree one in the variables $x$ and $y$. The Legendre transform is also a partly homogeneous function, but only with respect to $x$.

Consider the function

$f(x, y, u, w)=-\frac{x^{2}}{y}+u^{5} x+w^{2}$.

Since $f_{y}=\frac{\partial f}{\partial y}=\frac{x^{2}}{y^{2}}$, and therefore $y=\frac{x}{f_{y}^{1 / 2}}$, the Legendre transformation of $f$ with respect to $y$ yields

$$
\begin{aligned}
f_{2}\left(x, f_{y}, u, w\right) & =-\frac{x^{2} f_{y}^{1 / 2}}{x}+u^{5} x+w^{2}-\frac{x}{f_{y}^{1 / 2}}\left(\frac{x^{2} f_{y}}{x^{2}}\right) \\
& =-2 x f_{y}^{1 / 2}+u^{5} x+w^{2} .
\end{aligned}
$$

Example \#12 The Gibbs free energy function $\left(G\left(T, p, n_{1}, \ldots, n_{m}\right)\right)$ is obtained from the internal energy function $\left(U=U\left(S, V, n_{1}, \ldots, n_{m}\right)\right) \quad$ via appropriate Legendre transformations as

$G=U-T S+p V$,

where $S$ is the entropy, $V$ is the volume, $p$ is the pressure, $T$ is the temperature, and $n_{i}$ is the chemical amount of component i. $p$ and $T$ are given, respectively, as $p=-\frac{\partial U}{\partial V}$ and $T=\frac{\partial U}{\partial S}$. Note that the function $G$ is a typical example of a partly homogeneous function (see "The Gibbs-Duhem equation" section).

Acknowledgments The financial support from the Hungarian Scientific Research Fund (Grants No. K 109036) is gratefully acknowledged.

\section{References}

1. Lang GG, Barbero C (2012) Laser techniques for the study of electrode processes. Springer, Heidelberg

2. Koenig FO (1934) J Phys Chem 38:111-128

3. Koenig FO (1934) J Phys Chem 38:339-363

4. Grahame DC, Whitney RB (1942) J Am Chem Soc 64:1548-1552

5. Graham DC (1947) Chem Rev 41:441-501

6. Mohilner DM (1962) J Phys Chem 66:724-726

7. Hansen RS (1962) J Phys Chem 66:410-415

8. Soffer A (1972) J Electroanal Chem 40:153-165

9. Mohilner DM (1966) The electrical double layer. In: Bard AJ (ed) Electroanalytical chemistry, vol 1. Marcel-Decker, New York

10. Defay R, Prigigine I, Bellemans A, Everett DH (1966) Surface tension and adsorption. Longmans, Bristol

11. Goodrich FC (1968) Trans Farad Soc 64:3403-3415

12. Parsons R (1984) Thermodynamic methods for the study of interfacial regions in electrochemical systems. In: Bockris JO'M, Conway BE, Yeager E (eds) Comprehensive treatise of electrochemistry, the double layer, vol 1. Plenum Press, New York

13. Everett DH (1987) Pure Appl Chem 59:45-52

14. Girault HHJ, Schiffrin DJ (1989) Electrochemistry of liquid/liquid interfaces. In: Bard AJ (ed) Electroanalytical Chemistry, vol 15. Marcel Dekker, New York

15. Damaskin BB, Petrii OA (2011) J Solid State Electrochem 15:1317-1334

16. Gutman EM (2014) J Solid State Electrochem 18:3217-3237

17. Láng G, Heusler KE (1995) J Electroanal Chem 391:169-179

18. Láng GG, Seo M, Heusler KE (2005) J Solid State Electrochem 9:347-353

19. Gibbs JW (1948) Collected works, vol I. Yale University Press, New Haven, pp 219-237

20. Guggenheim EA, Adam NK (1933) Proc R Soc Lond A139:218-236

21. Guggenheim EA (1950) Thermodynamics, 2nd edn. Interscience Publishers Inc, New York

22. Trasatti S, Parsons R (1986) Pure Appl Chem 58:437-454

23. Everett DH (1972) Manual of symbols and terminology for physicochemical quantities and units, appendix ii, definitions, 
terminology and symbols in colloid and surface chemistry, part I. Butterworths, London

24. Eriksson JC (1969) Surf Sci 14:221-246

25. Eriksson JC (1965) Ark Kemi 25:331-341

26. Linford RG (1978) Chem Rev 78:81-95

27. Randles JEB, Behr B (1972) J Electroanal Chem 35:389-404

28. Guggenheim EA (1940) Trans Farad Soc 36:397-412

29. Callen H (1985) Thermodynamics and an introduction to thermostatistics, 2nd edn. Wiley, New York

30. Láng G, Heusler KE (1994) J Electroanal Chem 377:1-7

31. Heusler KE, Láng G (1997) Electrochim Acta 42:747-756

32. Kudryavtsev LD (2002) Homogeneous function. In: Hazewinkel M (ed) Encyclopaedia of mathematics. Springer, Berlin
33. Courant R, Hilbert D (1966) Methods of mathematical physics, partial differential equations, vol II. Interscience Pubs., John Wiley \& Sons, New York

34. Miller DG (1963) J Chem Educ 40:648-649

35. Duhem P (1886) Le Potentiel Thermodynamique et Ses Applications à La Mécanique Chimique et à L'Etude des Phénomènes Electriques. A. Hermann, Paris

36. Annamalai K, Puri IK (2002) Advanced thermodynamics engineering, CRC series in computational mechanics and applied analysis. CRC Press, Boca Raton 